\title{
Geometric motives and the h-topology
}

\author{
Jakob Scholbach
}

Received: 12 December 2006 / Accepted: 1 May 2009 / Published online: 21 December 2011

(C) Springer-Verlag 2011

\begin{abstract}
The aim of this paper is to give a detailed proof of a comparison of Voevodsky's categories of geometric motives with and without transfers, respectively. The latter category is defined by means of h-topology introduced by Voevodsky, a topology essentially given by Zariski coverings, finite coverings and blowups.
\end{abstract}

Keywords Motives $\cdot \mathrm{h}$-Topology $\cdot$ Sheaves with transfers

Mathematics Subject Classification (2000) $\quad 14 \mathrm{~F} 42$

\section{Introduction}

The category of geometric motives [24] is roughly defined to be the localisation of the category of smooth correspondences with respect to the affine line and the Zariski topology. Before, Voevodsky had defined a category of motives using the h-topology [22]. The objective of this paper is to give a detailed proof of a theorem of Voevodsky stating the equivalence of these two concepts in the case of rational coefficients and a base field of characteristic zero.

We see two principal facets of this description: on the one hand it clarifies why the technique of proper descent, which is often applied by replacing some scheme by a proper hypercovering in order to resolve its singularities, fits into the motivic framework (e.g. in [9]). On the other hand, the theorem is a hint that correspondences, which make the handling of the several categories of motives technically intricate, should not be necessary in the case of rational coefficients.

We define a category of effective quasigeometric motives with rational coefficients as follows: Let Sch denote the category of schemes, by which we mean schemes of finite type over a field $k$. Let $\mathbb{Q}$ Sch be the $\mathbb{Q}$-linear hull of Sch (i.e., morphisms are given by $\mathbb{Q}$-linear combinations of morphisms of schemes) and let $\overline{\mathbb{Q S c h}}$ be its closure under countable direct sums. Finally let $\mathbf{K}^{-}(\overline{\mathbb{Q} \mathbf{S c h}})$ denote the homotopy category of bounded above complexes in

J. Scholbach $(\varangle)$

Mathematisches Institut, Universität Freiburg, Eckerstr. 1, 79104 Freiburg, Germany 
$\overline{\mathbb{Q} S c h}$. We define the category

$$
\mathbf{D M}_{\mathrm{gm}, \mathrm{h}}^{\mathrm{eff}}(\mathbb{Q}):=\mathbf{K}^{-}(\overline{\mathbb{Q}} \mathbf{S c h}) /\left\{\mathbb{A}^{1} \text {-complexes, h-coverings }\right\}
$$

of effective quasigeometric motives to be the localization of $\mathbf{K}^{-}(\overline{\mathbb{Q S C h}})$ with respect to the following complexes:

- $\mathbb{A}^{1}$-complexes: $X \times \mathbb{A}^{1} \rightarrow X, X$ smooth

- Čech nerves $\cdots \rightarrow U \times{ }_{X} U \rightarrow U \rightarrow X$ of h-coverings $U \rightarrow X$, for $U$ smooth, $X \in \mathbf{S c h}$.

The h-topology is essentially given by Zariski open coverings, finite surjective morphisms and blowups (Definition 1.5, Proposition 1.6). The subcategory $\mathbf{D M}_{\mathrm{gm}, \mathrm{h}}^{\mathrm{eff}}(\mathbb{Q})$ of bounded complexes in $\mathbb{Q}$ Sch is called category of effective geometric motives.

On the other hand, the category of effective geometric motives with transfers is defined via the category $\mathbb{Q}$ SmCor, consisting of smooth schemes over $k$ and morphisms given by finite correspondences, i.e. $\mathbb{Q}$-linear combinations of cycles $W \subset X \times Y$ finite over $X$. Again, we first define a quasigeometric category by

$$
\mathbf{D} \mathbf{M}_{\overline{\mathrm{gm}}}^{\mathrm{eff}}(\mathbb{Q}):=\mathbf{K}^{-}(\overline{\mathbb{Q} S m C o r}) /\left\{\mathbb{A}^{1} \text {-complexes, Mayer-Vietoris }\right\},
$$

where $\overline{\mathbb{Q} S m C o r}$ is the closure of $\mathbb{Q}$ SmCor under countable direct sums, $\mathbb{A}^{1}$-complexes are as above, and Mayer-Vietoris complexes are given by $U \cap V \rightarrow U \sqcup V \rightarrow U \cup V(U, V$ smooth). Its subcategory $\mathbf{D} \mathbf{M}_{\mathrm{gm}}^{\mathrm{eff}}(\mathbb{Q})$ of complexes in $\mathbf{K}^{\mathrm{b}}(\mathbb{Q} \mathbf{S m C o r})$ is Voevodsky's category of effective geometric motives with transfers.

Theorem 4.1 Let $k$ be of characteristic zero. Then there is an equivalence between the categories of effective geometric motives with and without transfers, respectively (with rational coefficients):

$$
\mathbf{D M}_{\mathrm{gm}}^{\mathrm{eff}}(\mathbb{Q}) \stackrel{\cong}{\longrightarrow} \mathbf{D} \mathbf{M}_{\mathrm{gm}, \mathrm{h}}^{\mathrm{eff}}(\mathbb{Q}) .
$$

This equivalence derives from an analogous equivalence of the quasigeometric variants. These equivalences extend to the non-effective motivic categories (see Definition 4.4 for their definition).

It should be pointed out that the above comparison is - using Neeman's compacity notion [16] - a formal corollary of the equivalence of the two categories of effective motivic complexes, which is the non-torsion part of [24, Theorem 4.1.12]. Our aim is to give a detailed proof. It closely follows the ideas developed by Voevodsky and coworkers [20,22,25], especially concerning sheaves with transfers and their cohomology comparison results. For example, the precursor for the embedding theorem (3.14) and its proof is [24, Theorem 3.2.6]. Overview of the proof and of the paper: A principal notion are presheaves with transfers. These are just contravariant functors $\mathbf{S m C o r} \rightarrow \mathbf{A b}$. A presheaf is called homotopy invariant, if the canonical map $F(X) \rightarrow F\left(X \times \mathbb{A}^{1}\right)$ is an isomorphism.

The general idea is to refine the topology to avoid correspondences. The h-topology is well suited for this task: firstly, every h-sheaf has a unique transfer structure (Proposition 2.2). Secondly, for an homotopy invariant presheaf with transfers with rational coefficients, its sheafifications and cohomology groups with respect to the Zariski-, Nisnevich-, étale, cdh-, qfh- or h-topology agree (Theorem 2.11).

These facts give an equivalence between the categories of motivic complexes with rational coefficients, with and without transfers, respectively (Theorem 3.7):

$$
\mathbf{D M}_{\mathrm{Nis}, \mathrm{tr}}^{\mathrm{eff}}(\mathbb{Q}) \cong \mathbf{D} \mathbf{M}_{\mathrm{h}}^{\mathrm{eff},-}(\mathbb{Q}),
$$


where the former category is the full subcategory of the derived category of Nisnevich sheaves with transfers $\mathbf{D}^{-}\left(\mathbf{S h v}_{\mathrm{Nis}}\right.$, tr $\left.(\mathbf{S m})\right)$ consisting of complexes with homotopy invariant cohomology-Nisnevich-sheaves and the latter is defined similarly using h-sheaves instead.

Applying the cohomology comparison results, we obtain the embedding theorem (3.14):

$$
\mathbf{D M}_{\mathrm{gm}, \mathrm{h}}^{\mathrm{eff}}(\mathbb{Q}) \subset \mathbf{D} \mathbf{M}_{\mathrm{gm}, \mathrm{h}}^{\mathrm{eff}}(\mathbb{Q}) \subset \mathbf{D} \mathbf{M}_{\mathrm{h}}^{\mathrm{eff},-}(\mathbb{Q}) .
$$

The same statement is known for the situation with transfers (Voevodsky, see Theorem 3.15). More precisely, in either the situation with or without transfers, the geometric category is the subcategory of compact objects and the quasigeometric category is the one of $\aleph_{1}$-compact objects (Definition A.3). This completes the proof.

We restrict to characteristic zero in order to use resolution of singularities (see Proposition 1.1). We work with $\mathbb{Q}$-coefficients in order to ensure the finiteness of the cohomological dimension and the equality of Nisnevich and étale cohomologies. Another technically important point is Lemma 2.6, which is used to close the gap-essentially consisting of finite surjective morphisms - between Nisnevich and qfh-topology. Analogous cohomology comparison results for sheaves with integral coefficients are not true (see Remark 2.12).

\section{Notations and preliminary results}

We fix the following notations. The base field is denoted by $k$. By a scheme, we always mean a separated scheme of finite type over Spec $k$. The category of schemes is denoted Sch, its subcategory of smooth schemes is denoted $\mathbf{S m} ; \mathbb{Z} \mathbf{S c h}$ is the category whose objects are schemes, but morphisms are given by $\operatorname{Hom}_{\mathbb{Z} \mathbf{S c h}}(X, Y):=\bigoplus_{i} \mathbb{Z}\left[\operatorname{Hom}_{\mathbf{S c h}}\left(X_{i}, Y\right)\right]$, where $X_{i}$ are the connected components of $X$. By formally adding a zero object, we obtain an additive category.

Recall the definition of Voevodsky's category of finite correspondences SmCor (see, for example [15, esp. Lecture 1]): its objects are smooth schemes and its morphisms are defined by

$\operatorname{Hom}_{\operatorname{SmCor}}(X, Y):=\mathbb{Z}[W \subset X \times Y, \mathrm{~W}$ irreducible, surjective and finite over $X]$,

if $X$ is connected and $\operatorname{Hom}_{S m C o r}\left(\sqcup X_{i}, Y\right):=\oplus \operatorname{Hom}_{\operatorname{SmCor}}\left(X_{i}, Y\right)$ otherwise. The composition of correspondences is defined in the usual way (see loc. cit.). We obtain an additive category. Assigning to a morphism in $\mathbf{S m}$ its graph gives a faithful functor $\mathbf{S m} \rightarrow \mathbf{S m C o r}$ (see $[4, \S 16]$ for associativity of the composition of correspondences and functoriality).

Categories of presheaves are denoted by $\mathbf{P S h}(-)$, where - is either $\mathbf{S m}$ or $\mathbf{S c h}$. If not mentioned explicitly otherwise, presheaves will be presheaves of abelian groups. All presheaves will be understood to be additive, i.e. $F(X \sqcup Y) \cong F(X) \oplus F(Y)$. If $t$ is a topology, $t$-sheaves are denoted by $\mathbf{S h v}_{t}(-)$. The $t$-sheafification of a presheaf $F$ is denoted by $F_{t}$. In general we add a subscript - tr when there is some category or object "with transfers": the categories of $t$-sheaves with transfers (i.e. contravariant functors from SmCor to abelian groups whose restriction to $\mathbf{S m}$ is a $t$-sheaf) are denoted by $\mathbf{S h} \mathbf{v}_{t, \text { tr }}(\mathbf{S m})$ and similarly for presheaves with transfers: $\mathbf{P S h}_{\mathrm{tr}}(\mathbf{S m})$.

The representable presheaves on schemes are written $\mathbb{Z}(-)$ (and $\mathbb{Z}_{\mathrm{tr}}(-)$ for correspondences, i.e. $\left.\mathbb{Z}_{\mathrm{tr}}(X)(V)=\operatorname{Hom}_{\operatorname{SmCor}}(V, X)\right)$.

The $\mathbb{Q}$-rational variants of these constructions are denoted by $\mathbb{Q}$ Sch, $\mathbb{Q S m C o r}$, $\mathbb{Q}_{\text {tr }}, \mathbf{P S h}(\mathbf{S c h}, \mathbb{Q}), \mathbf{S h v}_{\mathrm{Nis}, \mathrm{tr}}(\mathbf{S m}, \mathbb{Q})$ etc.

Let Com* $(-), * \in\{-, \mathrm{b}\}$ denote the category of bounded above and bounded complexes, respectively (with differentials having degree +1$), \mathbf{K}^{*}(-)$ the corresponding homotopy category and $\mathbf{D}^{*}(-)$ the derived category. The total complex of a double complex is denoted 
by $\operatorname{Tot}(-)$ (see $[26,1.2 .6]$ for choice of signs). If $\mathcal{C}, \mathcal{D}$ are categories, $\mathcal{C} \subset \mathcal{D}$ denotes a full embedding.

We say " $k$ admits weak resolution of singularities", if every scheme $X$ admits a proper surjective morphism $Y \longrightarrow X$, where $Y$ is smooth.

We say " $k$ admits strong resolution of singularities", if furthermore for any abstract blowup $p: Y \longrightarrow X$ with nowhere dense closed center $Z \subset X$ of any scheme $X$ (i.e. any proper surjective map, such that $\left.p^{-1}(X-Z) \cong X-Z\right)$ there is a finite composition of blow-ups of smooth centers $q: \widetilde{X}=X_{n} \rightarrow \cdots \rightarrow X_{0}=X$ factoring through $p$.

Proposition 1.1 Any field admits weak resolution of singularities. Any field of characteristic 0 admits strong resolution of singularities.

Proof For the weak resolution, see [10, Main Theorem I, S. 132] (chark = 0) and [3, Theorem $4.1 ; \S 1](\operatorname{char} k>0)$.

For the strong resolution over a field of characteristic zero one applies the resolution of indeterminacies to $p^{-1}$, see [11] in the analytical setting and [1, §1.2.4], [17, Corollaire 5.7.12], [2, §1.2] for the algebraic setting.

Definition 1.2 For a simplicial scheme $\mathcal{X}$ we denote by $\mathbb{Z}(\mathcal{X})$ the Moore complex of representable presheaves

$$
\cdots \longrightarrow \mathbb{Z}\left(\mathcal{X}_{2}\right) \stackrel{\delta_{2}}{\longrightarrow} \mathbb{Z}\left(\mathcal{X}_{1}\right) \stackrel{\delta_{1}}{\longrightarrow} \mathbb{Z}\left(\mathcal{X}_{0}\right),
$$

where $\delta_{n}=\sum_{i=0}^{n+1}(-1)^{i} \partial_{n}^{i}$ and $\partial_{n}^{i}: \mathcal{X}_{n} \rightarrow \mathcal{X}_{n-1}$ is the $i$ th boundary map of $\mathcal{X}$.

For a morphism $U \rightarrow X$ set $U_{X}^{n}:=U \times_{X} \cdots \times_{X} U$ ( $n$ factors). We denote by $\mathcal{U}_{X}$ the simplicial scheme $\cdots \rightarrow U_{X}^{3} \rightarrow U_{X}^{2} \rightarrow U$ with the obvious boundary and degeneracy maps. If $U \rightarrow X$ is a covering with respect to a (pre-)topology $t, \mathcal{U}_{X}$ is a $t$-hypercovering of $X[19$, Exp. V, Def. 7.3.1.1.].

Lemma 1.3 Let $\mathcal{X}=\left(X_{n}\right)_{n \in \mathbb{N}}$ be a hypercovering in $(\mathbf{S c h} / X)_{t}, t$ an arbitrary topology. The augmented Moore complex of the corresponding representable $t$-sheaves $\mathbb{Z}_{t}(\mathcal{X})$ is exact:

$$
\cdots \rightarrow \mathbb{Z}_{t}\left(\mathcal{X}_{2}\right) \rightarrow \mathbb{Z}_{t}\left(\mathcal{X}_{1}\right) \rightarrow \mathbb{Z}_{t}\left(\mathcal{X}_{0}\right) \rightarrow \mathbb{Z}_{t}(X) \rightarrow 0
$$

Proof A theorem of Verdier [19, Exposé V, Théoréme 7.3.2(3)] asserts that for any hypercovering $\mathcal{X}$ in $(\mathbf{S c h} / X)_{t}$ the sheafification of the following complex is exact:

$$
\cdots \rightarrow \mathbb{Z}_{X}\left(\mathcal{X}_{2}\right) \rightarrow \mathbb{Z}_{X}\left(\mathcal{X}_{1}\right) \rightarrow \mathbb{Z}_{X}\left(\mathcal{X}_{0}\right) \rightarrow \mathbb{Z}_{X}(X)=\mathbb{Z} \rightarrow 0,
$$

where $\mathbb{Z}_{X}(U)(V):=\mathbb{Z}\left[\operatorname{Hom}_{S c h / X}(V, U)\right]$. The proof of loc. cit. is valid in our situation, if we replace $\mathbb{Z}_{X, t}(-)$ by $\mathbb{Z}_{\text {Spec } k, t}(-)=\mathbb{Z}_{t}(-)$.

Lemma 1.4 Let $f: U \rightarrow X$ be an arbitrary morphism. Then the complex

$$
\mathbb{Z}\left(\mathcal{U}_{X}\right) \rightarrow \mathbb{Z}(X) \rightarrow \operatorname{coker} \mathbb{Z}(f) \rightarrow 0
$$

is an exact complex (of presheaves).

Proof This is [22, Proposition 2.1.4]. We give a proof for the convenience of the reader. Let $A$ be an arbitrary connected scheme. For brevity, $(A,-)$ and $[A,-]$ denote $\operatorname{Hom}_{\operatorname{Sch}}(A,-)$ and $\mathbb{Z}(-)(A)=\operatorname{Hom}_{\mathbb{Z} \mathbf{S c h}}(A,-)$, respectively. We have to show that the following complex is exact:

$$
\cdots \rightarrow\left[A, U_{X}^{3}\right] \rightarrow\left[A, U_{X}^{2}\right] \rightarrow[A, U] \rightarrow[A, X] \rightarrow \operatorname{coker} \mathbb{Z}(f)(A) \rightarrow 0 .
$$


We may suppose $(A, U) \neq \emptyset$. Fix an arbitrary element $* \in(A, U)$.

Consider the simplicial set

$$
\left(A, \mathcal{U}_{X}\right): \cdots \rightarrow\left(A, U_{X}^{3}\right) \rightarrow\left(A, U_{X}^{2}\right) \rightarrow(A, U) .
$$

The very definition of homotopy groups [6, I.7.] $p_{n}:=\pi_{n}\left(\left(A, \mathcal{U}_{X}\right), *\right)$ and the universal property of the fiber product yields $p_{n}=0$ for $n \geq 1$. The homotopy set $p_{0}$ is a quotient of $(A, U)$ : morphisms $f, g: A \rightarrow U$ are identified iff there is a morphism $h: A \rightarrow U \times{ }_{X} U$ such that $f=p r_{1} \circ h, g=p r_{2} \circ h$. For the simplicially constant complex $\mathcal{Q}=\left(p_{0}\right)_{n \geq 0}$ we obviously have $\pi_{n}(\mathcal{Q})=0$ for $n \geq 1, \pi_{0}(\mathcal{Q})=p_{0}$. Thus, the canonical morphism of simplical sets $\left(A, \mathcal{U}_{X}\right) \rightarrow \mathcal{Q}$ is a weak equivalence in the sense of [6, I.7., page 32]. Weak equivalences are preserved by $\mathbb{Z}(-)$, thus the associated morphism of Moore complexes $\left[A, \mathcal{U}_{X}\right] \rightarrow \mathbb{Z}(\mathcal{Q})$ is a quasi-isomorphism [6, Proposition III.2.16., Corollary III.2.7.]. So, the complex is exact at $\left[A, U_{X}^{n}\right], n>1$.

Exactness at $[A, U]$ follows from the definition of the fiber product: Let $s=\sum_{i \in I} n_{i} \cdot s_{i}$ be mapped to 0 under $f$. One inductively reduces to the case \#I=2. Then it is obvious that there is $g \in\left[A, U_{X}^{2}\right]$, such that $g \mapsto s$.

\subsection{H-topologies}

Definition 1.5 [22] A morphism of schemes $f: X \rightarrow Y$ is called a topological epimorphism, if the topological space associated to $Y$ is a quotient topological space of $X$, i.e. if $f$ is surjective and $A \subset X$ is open iff $f(A) \subset Y$ is open. $f$ is called a universal topological epimorphism, if this holds for any base change.

Coverings of the h-topology on $\mathbf{S c h}$ are by definition finite families $\left\{f_{i}: U_{i} \rightarrow X\right\}$ of morphisms of finite type, such that $\sqcup f_{i}: \sqcup U_{i} \rightarrow X$ is a universal topological epimorphism. If the $f_{i}$ are furthermore quasi-finite, this family is defined to be a covering of the qfh-topology.

The $c$ dh-topology is the minimal Grothendieck topology containing Nisnevich coverings and abstract blow-ups (see above).

Normal qfh-coverings $\left\{f_{i}: U_{i} \rightarrow X\right\}$ are qfh-coverings having a factorization $f_{i}=f \circ j_{i}$, where $\left\{j_{i}: U_{i} \rightarrow U\right\}$ is a Zariski covering by open subsets and $f: U \rightarrow X$ is a finite surjective morphism.

A family of morphisms $\left\{f_{i}: U_{i} \rightarrow X\right\}$ is called normal h-covering, if it has a factorization $f_{i}=b \circ f \circ j_{i}$, where $\left\{j_{i}: U_{i} \rightarrow U\right\}$ is an open covering, $f: U \rightarrow X_{Z}$ a finite surjective map and $b: X_{Z} \rightarrow X$ the blow-up of $X$ in a closed, nowhere dense center $Z$.

Any scheme is h-locally smooth by Proposition 1.1. We have the following inclusions of topologies on Sch: Zar $\subseteq$ Nis $\subseteq$ ét $\subseteq$ qfh $\subseteq$ h and Nis $\subseteq$ cdh $\subseteq$ h.

Proposition 1.6 ([22, Theorem 3.1.9], [20, Lemma 10.4]) Every h-covering of a scheme admits a normal refinement. The same is true for qfh-coverings of normal schemes.

Lemma 1.7 Let $\mathcal{A}$ be an abelian category closed under countable direct sums. Let $\mathcal{B} \subset$ $\mathcal{D}:=\mathbf{D}^{-}(\mathcal{A})$ be a full triangulated subcategory closed under existing countable direct sums (i.e. $\aleph_{1}$-localising in the sense of Definition A.3). If all components $F^{n}$ of some complex $F \in \mathcal{D}$ are in $\mathcal{B}$, then $F$ is in $\mathcal{B}$.

Proof Compare [15, Lemma 9.4.].

We write $\mathcal{D}^{\prime}:=\mathbf{D}^{-}(\mathbf{P S h}(\mathbf{S c h}))$ and $\mathcal{D}:=\mathbf{D}^{-}\left(\mathbf{S h v}_{\mathrm{h}}(\mathbf{S c h})\right)$ in the following lemmas. The localising subcategory generated by a class of objects $T$, i.e. the minimal triangulated subcategory closed under existing direct sums containing $T$ will be denoted by $\langle T\rangle$ (see Definition A.2). 
Lemma 1.8 We have $\left\langle\mathbb{Z}_{\mathrm{h}}(X), X \in \mathbf{S m}\right\rangle=\mathcal{D}$ and $\langle\mathbb{Z}(X), X \in \mathbf{S c h}\rangle=\mathcal{D}^{\prime}$.

Proof For any h-sheaf $F$, the morphism $\bigoplus_{X \in \mathbf{S m}, a \in F(X)} \mathbb{Z}_{\mathrm{h}}(X) \stackrel{\oplus a}{\longrightarrow} F$ is surjective in $\mathbf{S h v}_{\mathrm{h}}(\mathbf{S c h})$ because of weak resolution of singularities. This easily yields the assertion (Lemma 1.7, applied to $\mathcal{B}=\left\langle\mathbb{Z}_{\mathrm{h}}(X), X \in \mathbf{S m}\right\rangle$ ). The statement for presheaves is proven similarly.

Lemma 1.9 Let $T_{\mathrm{h}} \subset \mathcal{D}^{\prime}$ denote the class of augmented Moore complexes

$$
\cdots \rightarrow U \times{ }_{X} U \rightarrow U \rightarrow X
$$

of h-coverings $U \rightarrow X$, where $U \in \mathbf{S m}, X \in \mathbf{S c h}$ (Definition 1.2). The sheafification functor $\mathcal{D}^{\prime} \rightarrow \mathcal{D}$ factorizes over $\mathcal{D}^{\prime} /\left\langle T_{\mathrm{h}}\right\rangle$ and induces an equivalence of categories $\mathcal{D}^{\prime} /\left\langle T_{\mathrm{h}}\right\rangle \cong \mathcal{D}$.

Proof Let $\mathcal{C}_{\mathrm{h}}$ denote the localising subcategory of $\mathcal{D}^{\prime}$ generated by presheaves $F$ with $F_{\mathrm{h}}=0$. With Lemma 1.7, it is quite obvious to see that we have $\mathcal{D} \cong \mathcal{D}^{\prime} / \mathcal{C}_{\mathrm{h}}$, so it suffices to show $T_{\mathrm{h}}=\mathcal{C}_{\mathrm{h}}$. We have $\left\langle T_{\mathrm{h}}\right\rangle \subseteq \mathcal{C}_{\mathrm{h}}($ Lemma 1.3).

For the converse, choose a complex $F \in \mathcal{C}_{\mathrm{h}}$. We may assume that it is concentrated in degree 0 (Lemma 1.7). Therefore, there is an epimorphism of presheaves $\oplus_{i} \operatorname{coker} \mathbb{Z}\left(f_{i}\right) \rightarrow$ $F$, where $f_{i}: U_{i} \rightarrow X_{i}$ are h-coverings. Using weak resolution of singularities we may assume that the $U_{i}$ are smooth. As (coker $\left.\mathbb{Z}\left(f_{i}\right)\right)_{\mathrm{h}}=0$, we even get a resolution of $F$ by sheaves of this form. We may thus assume $F=$ coker $f$ for an h-covering $f: U \rightarrow X$ (Lemma 1.7). The sequence

$$
\cdots \rightarrow \mathbb{Z}\left(U \times_{X} U\right) \rightarrow \mathbb{Z}(U) \longrightarrow \mathbb{Z}(X) \longrightarrow \operatorname{coker} f \longrightarrow 0
$$

is exact (Lemma 1.4). The augmented Moore complex lies in $\left\langle T_{\mathrm{h}}\right\rangle$ and so does $F=\operatorname{coker} f$.

Remark 1.10 The proof shows that the proposition still holds if we enlarge $T_{\mathrm{h}}$ by adding complexes becoming exact when they are h-sheafified. In particular one may add arbitrary h-hypercoverings (Lemma 1.3). On the other hand, it suffices to localise with respect to h-coverings $\mathbb{Z}\left(\mathcal{U}_{X}\right) \rightarrow \mathbb{Z}(X)$, where $U \rightarrow X$ is of normal form (Proposition 1.6).

\section{Sheaves with transfers}

We are going to study the relationship between sheaves with and without transfers. It turns out that every h-sheaf has a unique transfer structure. Note that the sheafification of a presheaf with transfers is in general (i.e. for an arbitrary topology) not necessarily a presheaf with transfers.

Lemma 2.1 The canonical (mono-)morphisms $\mathbb{Z}(X) \rightarrow \mathbb{Z}_{\mathrm{tr}}(X), X \in \mathbf{S m}$ induce isomorphisms

$$
\mathbb{Z}_{\mathrm{h}}(X) \cong \mathbb{Z}_{\mathrm{tr}, \mathrm{h}}(X), \quad \mathbb{Q}_{\mathrm{h}}(X) \cong \mathbb{Q}_{\mathrm{tr}, \mathrm{h}}(X) .
$$

They induce a transfer structure on any $h$-sheaf. We call it the canonical transfer structure.

Proof The second part of the lemma is stated by Voevodksy [23, §3.4.]. The lemma was also proven independently by Ivorra [13, Corollaire 3.2.19].

Fix a smooth scheme $V$. By definition, $c(X / k, 0)(V)=c\left(X \times_{k} V / V, 0\right)$ is the group of universally integral cycles of $X \times_{k} V$, whose support is proper of relative dimension 0 over $V[21, \S 2.3 ., 3.1 .1-3.1 .3$, definition after Lemma 3.3.9]. This presheaf is obviously additive. Since all presheaves we are dealing with are additive, we may suppose that $V$ is connected. 
To be proper of relative dimension 0 is the same as to be finite over $V$. As $V$ is regular, any relative cycle is universally integral [21, Proposition 3.3.15]. $V$ is normal, thus geometrically "unibranche" $[8,6.15 .1,0.23 .2 .1]$, therefore any subset $Z \subseteq X$ which is equidimensional over $V$ defines a relative cycle [21, Theorem 3.4.2].

$$
c(X / k, 0)(V) \supset \mathbb{Z}\left[\begin{array}{l}
Z \subset X \times V, \quad \text { finite over } \\
V, \quad \text { equidimensional over } V
\end{array}\right]
$$

Any effective cycle is equidimensional [21, Proposition 3.1.7]. Thus we obtain for any smooth connected $V$ :

$$
c^{\text {eff }}(X / k, 0)(V)=\mathbb{N}[Z \subseteq X \times V, Z \text { finite over } V, Z \text { irred. }]=: \mathbb{N}_{\operatorname{tr}}(X)(V),
$$

i.e. $c^{\text {eff }}(X / k, 0)(V)=\operatorname{Hom}_{\mathbb{N S m C o r}}(V, X)$ (the free abelian monoid generated by elementary correspondences from $V$ to $X)$. The functoriality of the two presheaves agrees [21, Corollary 3.3.11]. We obtain an equality of presheaves on $\mathbf{S m}:\left.c^{\mathrm{eff}}(X / k, 0)\right|_{\mathbf{S m}}=\mathbb{N}_{\mathrm{tr}}(X)$, and a fortiori $c_{\mathrm{h}}^{\text {eff }}(X / k, 0)=\mathbb{N}_{\text {tr }, \mathrm{h}}(X)$. Moreover, the map $\mathbb{N}(X) \rightarrow c^{\text {eff }}(X / k, 0)$ mapping a morphism of schemes to its graph induces an isomorphism $\mathbb{N}_{\mathrm{h}}(X) \stackrel{\cong}{\longrightarrow} c_{\mathrm{h}}^{\text {eff }}(X / k, 0)[21$, Theorem 4.2.12(2)].

Assembling these isomorphisms together we conclude that the canonical monomorphism $\mathbb{N}(X) \rightarrow \mathbb{N}_{\text {tr }}(X)$ becomes an isomorphism when we apply h-sheafification. This yields the assertions for $\mathbb{Z}_{\text {tr }}$ and $\mathbb{Q}_{\text {tr }}$ as well.

The canonical transfer structure is given as follows: let $F$ be an h-sheaf (without transfers), $X \in \mathbf{S m}$. Then we have

$$
\begin{aligned}
F(X) & =\operatorname{Hom}_{\mathbf{P S h}(\mathbf{S m})}(\mathbb{Z}(X), F)=\operatorname{Hom}_{\mathbf{S h v}_{\mathrm{h}}(\mathbf{S m})}\left(\mathbb{Z}_{\mathrm{h}}(X), F\right) \\
& \cong \operatorname{Hom}_{\mathbf{S h v}_{\mathrm{h}}(\mathbf{S m})}\left(\mathbb{Z}_{\mathrm{tr}, \mathrm{h}}(X), F\right)=\operatorname{Hom}_{\mathbf{P S h}(\mathbf{S m})}\left(\mathbb{Z}_{\mathrm{tr}}(X), F\right) .
\end{aligned}
$$

Let $Z \in \operatorname{Hom}_{\mathbf{S m C o r}}(Y, X)=\operatorname{Hom}_{\mathbf{P S h}}(\mathbf{S m})\left(\mathbb{Z}_{\mathrm{tr}}(Y), \mathbb{Z}_{\mathrm{tr}}(X)\right)$. Now, $F(Z): F(X) \rightarrow F(Y)$ is defined using the above isomorphism by $a \mapsto a \circ \mathbb{Z}_{\mathrm{tr}}(Z)$. This clearly defines a transfer structure on $F$ whose restriction to $\mathbf{S m}$ coincides with the original h-sheaf on $\mathbf{S m}$.

Proposition 2.2 There is an equivalence of categories of $h$-sheaves with and without transfers:

$$
\operatorname{Shv}_{\mathrm{h}, \mathrm{tr}}(\mathbf{S m}) \cong \operatorname{Shv}_{\mathrm{h}}(\mathbf{S c h}) .
$$

Proof Note that h-sheaf on $\mathbf{S m C o r}$ (i.e. on $\mathbf{S m}$ ) is meant in the sense of the induced topology under the embedding $\mathbf{S m} \subset \mathbf{S c h}[18, \operatorname{Expos} § I I, 1.1 ., 3.1$.$] . As any scheme is h-locally$ smooth, we have an equivalence of h-sheaves on Sch and h-sheaves on Sm [18, Exposé III, Théoréme 4.1].

We make the following obvious remark: for any presheaf with transfers $G$ the following functors on $\mathbf{S m}$ are naturally isomorphic:

$$
G(-) \cong \operatorname{Hom}_{\mathbf{P S h}}(\mathbf{S m})\left(\mathbb{Z}_{\mathrm{tr}}(-), G\right) \cong \operatorname{Hom}_{\mathbf{P S h}(\mathbf{S m})}\left(\mathbb{Z}_{\mathrm{tr}}(-), G\right) .
$$

The first bijection is the Yoneda lemma. The second one is shown similarly using the transfer structure of $G$.

First, we show that for any presheaf with transfers $F$ the canonical morphism $F \rightarrow F_{\mathrm{h}}$ is a morphism of presheaves with transfers. $F_{\mathrm{h}}$ has its canonical transfer structure (Lemma $2.1)$, in particular we have $F_{\mathrm{h}}(X)=\operatorname{Hom}_{\mathbf{P S h}(\mathbf{S m})}\left(\mathbb{Z}_{\mathrm{tr}}(X), F_{\mathrm{h}}\right)$. For a correspondence $Z \in \operatorname{Hom}_{\text {SmCor }}(Y, X)$ the morphisms $F(X) \rightarrow F(Y)$ and $F_{\mathrm{h}}(X) \rightarrow F_{\mathrm{h}}(Y)$ are induced 
by the map $\mathbb{Z}_{\mathrm{tr}}(Y) \rightarrow \mathbb{Z}_{\mathrm{tr}}(X)$, by the Yoneda lemma and by construction of the canonical transfer structure, respectively. Consider the following diagram:

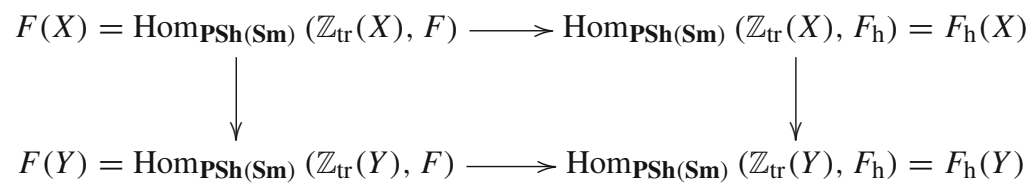

The horizontal maps are induced by morphisms of presheaves (without transfers) $F \rightarrow F_{\mathrm{h}}$. The commutativity of the diagram yields the first claim.

Secondly, one analogously proves that morphisms of h-sheaves are morphisms of hsheaves with transfers.

Thirdly, we show that any h-sheaf admits only one transfer structure. Considering any hsheaf $F$, the canonical morphism $F \rightarrow F_{\mathrm{h}}$ is an isomorphism of presheaves without transfer. According to the first claim, it is also a morphism of sheaves with transfers, where $F_{\mathrm{h}}$ carries the canonical transfer structure. This implies that $F$ carries the canonical transfer structure, as well.

\subsection{Cohomology comparison results}

Recall that a presheaf $F$ on $\mathbf{S c h}$ (or $\mathbf{S m}$ ) is called homotopy invariant, if the canonical projection $p: X \times \mathbb{A}^{1} \rightarrow X$ induces an isomorphism $p^{*}: F(X) \rightarrow F\left(X \times \mathbb{A}^{1}\right)$ for any $X$ in Sch (or Sm, respectively). One easily sees that for any complex $F^{*}$ of homotopy invariant presheaves the cohomology presheaves $\mathrm{H}^{n}\left(F^{*}\right)$ are homotopy invariant, as well.

First of all, we gather several results of Friedlander, Suslin and Voevodsky in the following theorem. These facts constitute the nontrivial input to the comparison result:

Theorem 2.3 Let $k$ be a field admitting strong resolutions of singularities. Let $F$ be a homotopy invariant presheaf of $\mathbb{Q}$-vector spaces with transfers. Let $t$ be either the Zariski, Nisnevich, étale or cdh-topology. Then the cohomology presheaves on $\mathbf{S m}$

$$
X \mapsto \mathrm{H}_{t}^{n}\left(X, F_{t}\right), \quad n \geq 0
$$

are homotopy invariant presheaves with transfers and are (up to canonical isomorphisms) independent of $t$.

Proof Presheaves with transfers are pretheories [24, Proposition 3.1.11]. Hence [23, Theorem 4.27, Proposition 5.28] (Zariski-topology), [23, Theorem 5.7.] (Nisnevich), [23, Propositions $5.24,5.27$ (étale topology, this requires rational coefficients) and finally the proof of [5, Theorem 5.5] (cdh-topology, this requires strong resolution of singularities) apply.

We want to generalize this theorem to the qfh- and h-topology. We first observe that the htopology is the "union" of the cdh- and the qfh-topology, in the sense made precise by Lemma 2.4 below. Looking at the definitions of these topologies and Proposition 1.6, this statement is not surprising. We then use the usual Galois descent method in order to overcome the gap between Nisnevich and qfh-topology (Lemma 2.6). The cohomology comparison result between étale and qfh-topology (for a qfh-sheaf, Theorem 2.7) and the adaptation of the cohomology comparison result of Nisnevich versus cdh-topology to the case qfh- versus h-topology then give the result.

Lemma 2.4 Let $F$ be a presheaf on $\mathbf{S c h}$. We assume that $F$ is a qfh-sheaf and a cdh-sheaf. Then $F$ is an h-sheaf. 
Proof Consider the exact sequence of presheaves

$$
0 \longrightarrow \mathrm{ker} \longrightarrow F \longrightarrow F_{\mathrm{h}} \longrightarrow \text { coker } \longrightarrow 0 .
$$

Sheafifying it with respect to either the cdh- or the qfh-topology does not affect the middle terms and shows that both ker and coker are cdh- and qfh-sheaves. Thus, we are reduced to show $F=0$ for any $F$ which is both a qfh- and cdh-sheaf satisfying $F_{\mathrm{h}}=0$.

Let $X \in \mathbf{S c h}$, without loss of generality $X$ is reduced (as $F$ is a qfh-sheaf), $a \in F(X)$. There is an h-covering $\left\{g_{i}: U_{i} \rightarrow X\right\}$, such that $g_{i}^{*}(a)=0$. We may assume that the covering is of normal form (Proposition 1.6):

$$
g_{i}: U_{i} \stackrel{f_{i}}{\longrightarrow} U \stackrel{f}{\longrightarrow} X_{X_{1}} \stackrel{p}{\longrightarrow} X .
$$

Clearly $\left\{U_{i} \stackrel{f \circ f_{i}}{\longrightarrow} X_{X_{1}}\right\}$ is a qfh-covering. Hence $p^{*}(a)=0$, for $F$ is a qfh-sheaf. Since $F$ is a cdh-sheaf and $X_{X_{1}} \sqcup X_{1} \rightarrow X$ is a cdh-covering, it suffices to show $F\left(X_{1}\right)=0$.

We proceed by noetherian induction with respect to the strictly descending chain of closed subsets $X=: X_{0} \supsetneq X_{1} \supsetneq \cdots$. The induction stops when the chain becomes stationary or if the normal refinement of the covering of $X_{i}$ does not contain any blow-up. The former is impossible, thus at some point of the noetherian induction the h-covering of $X_{i}$ must even be a qfh-covering.

Theorem 2.5 (Comparison of Nisnevich and (qf)h-sheafification) We assume that $k$ admits strong resolution of singularities. Let $F$ be a homotopy invariant presheaf of $\mathbb{Q}$-vector spaces with transfers (on $\mathbf{S m})$. Then the Nisnevich, qfh-and h-sheafifications $F_{\mathrm{Nis}}, F_{\mathrm{qfh}} \mid \mathrm{Sm}$ and $\left.F_{\mathrm{h}}\right|_{\mathrm{Sm}}$ are isomorphic.

Consider the presheaves $\mathbb{Q}_{t^{\prime}}(X) \quad[20, \S 5$ and $\S 6]$, where $X \in \mathbf{S c h}$ :

$$
\mathbb{Q}_{t^{\prime}}(X)(Y):=\mathbb{Q}\left[\begin{array}{l}
W \subset X \times Y, \text { closed, integral, } \\
\text { finite and surjective over } Y
\end{array}\right],
$$

where $Y$ is an arbitrary normal connected scheme. Analogously we have a presheaf $\mathbb{Z}[1 / p]_{\operatorname{tr}^{\prime}}(X)$, where $p$ is the exponential characteristic of $k$. Functoriality is given as follows: Let $f: Z \rightarrow Y$ be a morphism of normal connected schemes, $W \subset X \times Y$ an integral cycle, finite and surjective over $Y$. Set $f^{*}([W]):=\sum n_{i}\left[\left(V_{i}\right)_{\text {red }}\right]$, where $V_{i}$ are the irreducible components of $W \times_{Y} Z$ and $\left(V_{i}\right)_{\text {red }}$ are the associated reduced (thus integral) schemes. The multiplicities $n_{i}$ are defined as described in [20, definition after Lemma 5.6]. They are always positive, see loc. cit.

Lemma 2.6 Let $f: Y \longrightarrow X$ be the normalization of a smooth (thus excellent) connected affine scheme $X$ in a normal finite field extension $L$ of $k(X)$. Then there exists a correspondence

$$
f_{*} \in \mathbb{Z}\left[\frac{1}{p}\right]_{\mathrm{tr}^{\prime}}(Y)(X)
$$

(finite integral correspondence from $X$ to $Y$ with coefficients in $\mathbb{Z}[1 / p]$ ), such that $f \circ f_{*}=$ $d \cdot \operatorname{Id}_{X}, d \in \mathbb{N}^{>0}$.

Proof (compare [22, Theorem 3.3.8] for $\mathbb{Z}_{\mathrm{qfh}}$ ).

The automorphism group $G:=\operatorname{Aut}(Y / X)=\operatorname{Gal}(k(Y) / k(X))$ is a finite group. Considering $g \in G$ as the graph of the corresponding automorphism, the sum $a=\sum_{g \in G} g$ is a 
$G$-invariant element of $\mathbb{Z}_{\mathrm{tr}^{\prime}}(Y)(Y)(a$ is an integral cycle as $Y$ is integral). According to [20, Corollary 6.2], $a$ has a preimage $f_{*} \in \mathbb{Z}[1 / p]_{\mathrm{tr}^{\prime}}(Y)(X)$ :

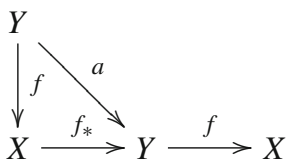

Let $d$ denote the cardinality of $G$. Then $f \circ f_{*} \circ f=f \circ a=d \cdot f$.

We show that for any surjective morphism $f: Y \rightarrow X$ of schemes, where $Y$ is assumed to be integral, and $X$ to be smooth and every effective correspondence $c \in \mathbb{N}_{\text {tr }^{\prime}}(X)(X)$ with $c \circ f=d \cdot f$ the equality $c=d \cdot \operatorname{Id}_{X}$ holds. This provides the assertion of the lemma, for $f$ is surjective in our situation and-by the proof of loc. cit. $-f_{*}$, being the preimage of an effective correspondence, is effective as well.

Let $c=\sum_{j} n_{j} \cdot c_{j}, n_{j} \in \mathbb{N}, c_{j} \subset X \times X$ integral and finite over $X$. By definition

$$
c \circ f=\sum_{i, j} n_{j} \cdot m_{i j} \cdot W_{i j} \stackrel{!}{=} d \cdot \Gamma_{f},
$$

where $W_{i j} \subset T_{j}$ are the associated reduced schemes of the irreducible components of $T_{j}:=c_{j} \times_{X} Y$, the morphism $c_{j} \rightarrow X$ is the projection to the first component. The multiplicities of the components $m_{i j}$ are positive (see above). As all coefficients are positive, it follows $W_{i j}=\Gamma_{f}$ for all $i, j$ and $\sum_{i, j} n_{j} m_{i j}=d$.

Using the surjectivity of $f$ it is easy to see (using Hilbert's Nullstellensatz) that the $\bar{k}$ valued points of $\Delta_{X}$ and $c_{j}$ agree. Thus $\Delta_{X}=c_{j}$ on the level of topological spaces (Hilbert's Nullstellensatz). As $X$, and thus $\Delta_{X}$ is reduced, they agree as schemes, too. Hence we have $\#\{j\}=1$ and $\#\{i\}=1$ as $T=\Gamma_{f} \cong Y$ is irreducible. Moreover $m=1$, for $Y$ is integral by assumption.

Proof of Theorem 2.5 In view of Theorem 2.3 we may assume that $F$ is an homotopy invariant cdh-sheaf with transfers, which implies that $F_{\mathrm{qfh}}$ is an h-sheaf (Lemma 2.4), i.e. $F_{\mathrm{qfh}}=F_{\mathrm{h}}$. Consider the exact sequence of Nisnevich sheaves with transfers:

$$
0 \longrightarrow F^{\prime} \longrightarrow F \longrightarrow F_{\mathrm{qfh}} \longrightarrow F^{\prime \prime} \longrightarrow 0 .
$$

(Recall that $\mathbf{S h} \mathbf{v}_{\mathrm{Nis}, \mathrm{tr}}(\mathbf{S m})$ is an abelian category [24, Theorem 3.1.4] and that $F \rightarrow F_{\mathrm{qfh}}=$ $F_{\mathrm{h}}$ is a morphism of sheaves with transfers, see Proposition 2.2).

As in the proof of Lemma 2.4 it suffices to show the following: Let $F$ be a (not necessarily homotopy invariant) cdh-sheaf on Sch, whose restriction to $\mathbf{S m}$ is a sheaf with transfers, such that $F_{\mathrm{qfh}}=0$. Then we have $F=0$.

Let $X \in \mathbf{S c h}$, without loss of generality affine and smooth (as $F$ is a cdh-sheaf), and $a \in F(X)$ an arbitrary section. We show $a=0$. There is a qfh-covering of normal form $\left\{f_{i}: U_{i} \rightarrow U \rightarrow X\right\}$, such that $f_{i}^{*}(a)=0$ (Proposition 1.6). As $F$ is a Zariski sheaf it suffices to consider $U \rightarrow X$. We replace the excellent scheme $U$ by its normalization in a normal finite extension of $k(X)$. Choose a cdh-covering $\widetilde{U} \rightarrow U$ with $\widetilde{U} \in \mathbf{S m}$ (strong resolution of singularities).

As $F$ is a sheaf with transfers,

$$
\begin{aligned}
F(V) & =\operatorname{Hom}_{\mathbf{P S h}}(\mathbf{S m})\left(\mathbb{Q}_{\mathrm{tr}}(V), F\right) \\
& =\operatorname{Hom}_{\mathbf{P S h}(\mathbf{S m})}\left(\mathbb{Q}_{\mathrm{tr}}(V), F\right) \\
& =\operatorname{Hom}_{\mathbf{P S h}(\mathbf{S m})}\left(\mathbb{Q}_{\mathrm{tr}}(V), F\right)
\end{aligned}
$$


for any smooth $V$ (see the proof of Proposition 2.2). As $F$ is a cdh-sheaf, we may cdhsheafify the first presheaf in all of these Hom-sets without changing the Hom-sets. Hence, the morphism $F(X) \rightarrow F(\widetilde{U})$ is induced by

$$
\mathbb{Q}_{\mathrm{tr}}, \operatorname{cdh}(\tilde{U}) \stackrel{\alpha}{\longrightarrow} \mathbb{Q}_{\mathrm{tr}^{\prime}, \operatorname{cdh}}(U) \stackrel{\beta}{\longrightarrow} \mathbb{Q}_{\mathrm{tr}^{\prime}, \mathrm{cdh}}(X) .
$$

We know $\left.\mathbb{Q}_{\text {tr' }}(W)\right|_{\mathbf{S m}}=\left.\mathbb{Q}_{\mathrm{qfh}}(W)\right|_{\mathbf{S m}}[20$, Theorem 6.3] for any scheme $W$. Thus, $a$ fortiori, $\mathbb{Q}_{\mathrm{tr}}(W)_{\mathrm{cdh}}=\mathbb{Q}_{\mathrm{qfh}, \mathrm{cdh}}(W)=\mathbb{Q}_{\mathrm{h}}(W)$. As $\widetilde{U} \rightarrow U$ is an h-covering, $\alpha$ is an epimorphism [22, Proposition 3.2.5.2]. According to Lemma 2.6, $\beta$ has a section, so it is an epimorphism. Hence $F(X) \rightarrow F(\widetilde{U})$ is a monomorphism, i.e. $a=0$.

Theorem 2.7 (Comparison of etale and qfh-cohomology) We assume that $k$ admits strong resolution of singularities. Let $F$ be a homotopy invariant étale sheaf with transfers with values in $\mathbb{Q}$-vector spaces and let $X$ be a normal scheme. Then $\mathrm{H}_{\mathrm{et}}^{n}(X, F) \cong \mathrm{H}_{\mathrm{qfh}}^{n}\left(X, F_{\mathrm{qfh}}\right), n \in \mathbb{N}$.

Proof The case $n=0$ is Theorem 2.5, so we may assume that $F$ is a qfh-sheaf. Then [22, Theorem 3.4.1] is what we assert.

Theorem 2.8 (Comparison of h- and qfh-cohomology) Let $k$ be a field admitting strong resolution of singularities and let $F$ be a homotopy invariant presheaf with transfers of $\mathbb{Q}$-vector spaces. Then for all $X \in \mathbf{S m}, n \geq 0$ the following holds:

$$
\mathrm{H}_{\mathrm{h}}^{n}\left(X, F_{\mathrm{h}}\right)=\mathrm{H}_{\mathrm{qfh}}^{n}\left(X, F_{\mathrm{qfh}}\right) .
$$

Lemma 2.9 Let $X \in \mathbf{S m}, Z \subset X$ a smooth closed subscheme. Let $p_{Z}: X_{Z} \rightarrow X$ denote the blow-up of $Z$ in $X$. Further, let $F$ be a homotopy invariant presheaf with transfers of $\mathbb{Q}$-vector spaces. Then it holds for $i \geq 0$

$$
\left.\operatorname{Ext}_{\mathbf{S h v}_{\mathrm{qfh}}(\mathbf{S m})}^{i}\left(\operatorname{coker} \mathbb{Z}_{\mathrm{qfh}}\left(p_{Z}\right)\right), F_{\mathrm{qfh}}\right)=0 .
$$

Proof An analogous statement for the Nisnevich topology is proven in [23, Proposition 5.21] exploiting the fact that Nisnevich cohomology groups of $F_{\mathrm{Nis}}$ are homotopy invariant. The proof of loc. cit. remains valid for any finer topology $t$, which fulfills $\mathrm{H}_{t}^{i}\left(X, F_{t}\right) \cong$ $\mathrm{H}_{t}^{i}\left(X \times \mathbb{A}^{1}, F_{t}\right)$. In particular it holds (for sheaves of $\mathbb{Q}$-vector spaces) for the qfh-topology (Theorem 2.3, Theorem 2.7).

Lemma 2.10 Let $k$ be a field admitting strong resolution of singularities, $F$ a qfh-sheaf, such that $F_{\mathrm{h}}=0, G$ a homotopy invariant presheaf with transfers of $\mathbb{Q}$-vector spaces. Then we have

$$
\operatorname{Ext}_{\mathbf{S h v}_{\mathrm{qfh}}(\mathbf{S m})}^{n}\left(F, G_{\mathrm{qfh}}\right)=0, \quad n \geq 0 .
$$

Proof The proof of [5, Lemma 5.4] goes through (using Lemma 2.9 instead of [5, Lemma 5.3]), if we replace Nisnevich and cdh-topology by qfh- and h-topology respectively.

Proof of Theorem 2.8 The proof of [5, Theorem 5.5] goes through verbatim using Lemma 2.10 instead of [5, Lemma 5.4].

The following theorem gathers the above results. It points out the quite special nature of homotopy invariant Zariski sheaves with transfers of $\mathbb{Q}$-vector spaces: they are already sheaves with respect to the (much finer) h-topology. 
Theorem 2.11 Let $t \in\left\{\mathrm{Zar}, \mathrm{Nis}\right.$, ét, cdh, qfh, h\}. Let $F^{*}$ be a bounded above complex of presheaves of $\mathbb{Q}$-vector spaces with transfers, whose cohomology-t-sheaves are homotopy invariant. We assume that $k$ admits strong resolution of singularities. Then the hypercohomology presheaves $X \mapsto \mathbb{H}_{t}^{n}\left(X, F_{t}^{*}\right), X \in \mathbf{S m}$ are homotopy invariant presheaves with transfers and are independent of the choice of the topology $t$.

The assumption on $F^{*}$ is satisfied, if the cohomology presheaves of $F^{*}$ are homotopy invariant.

Proof If $F^{*}$ is concentrated in degree 0, we are done because of Theorem 2.3, Theorem 2.7, Theorem 2.5, Theorem 2.8, as hypercohomology is just cohomology in this case. This also shows the last claim.

In general, let denote $\left(\mathrm{H}^{q}\left(F^{*}\right)\right)_{t}$ the $t$-sheafification of the $q$ th cohomology presheaf of the complex $F^{*}$, which is an homotopy invariant presheaf with transfers. The presheaves $X \mapsto \mathbb{H}_{\mathrm{Nis}}^{n}\left(X, F_{\mathrm{Nis}}^{*}\right)$ have transfers [15, Exercise 13.5.]. So, the other hypercohomology groups, which are canonically isomorphic to the Nisnevich hypercohomology, have transfers as well. The hypercohomology spectral sequence

$$
E_{2}^{p, q}=\mathrm{H}_{t}^{p}\left(X,\left(\mathrm{H}^{q}\left(F^{*}\right)\right)_{t}\right)=\operatorname{Ext}^{p}\left(\mathbb{Q}_{t}(X),\left(\mathrm{H}^{q}\left(F^{*}\right)\right)_{t}\right) \Longrightarrow \mathbb{H}_{t}^{p+q}\left(X, F_{t}^{*}\right)
$$

converges because of finite cohomological dimension for sheaves of $\mathbb{Q}$-vector spaces (see [22, Theorem 3.4.6] for the h-topology). Comparing the limit terms for $X$ and $X \times \mathbb{A}^{1}$ yields the assertion.

Remark 2.12 We restrict to $\mathbb{Q}$-linear categories as the comparison of Nisnevich and qfhsheaves is valid only for sheaves of $\mathbb{Q}$-vector spaces. An analogous statement for sheaves with torsion does not hold: Consider the Nisnevich sheaf with transfers $\mathcal{O}^{*}$. If $R$ is a ring, then $\mathcal{O}^{*}(R[t])=R^{*} \times\left\{1+\sum_{i=1}^{n} f_{i} t^{i},\left(f_{i}\right)^{N}=0, N \gg 0\right\}$. In particular, $\mathcal{O}^{*}$ is homotopy invariant on Sm, but not on Sch. See [23, Remark 5.8] for a counterexample for the Nisnevichflat topology.

The restriction to characteristic zero is only needed for the cohomology comparison result of Nis- and cdh-topology (and the analogue qfh- vs. h-topology). It seems reasonable to aim at a generalization to positive characteristic at least for $\mathbb{Q}$-coefficients using Noether normalization and de Jong's resolution of singularities, but we did not succeed to prove it.

\section{Motivic complexes and geometric motives}

\subsection{Motivic complexes}

As we have outlined in the introduction, we will embed the several categories of geometric motives - which we are mostly interested in-into the more flexible categories of motivic complexes. Voevodsky defines a category of motivic complexes using Nisnevich-sheaves with transfers. Parallely, we also use a different version using h-sheaves without transfers [22]. In the case of $\mathbb{Q}$-vector space sheaves both categories are equivalent (Theorem 3.7). This theorem relies on the cohomology comparison results of 2 .

Definition 3.1 [24, §3.1.] The category of effective motivic complexes with transfers is defined to be the full subcategory $\mathbf{D M}_{\mathrm{Nis} \text {,tr }}^{\text {eff, }}$ of $\mathbf{D}^{-}\left(\mathbf{S h v}_{\mathrm{Nis}, \text { tr }}(\mathbf{S m})\right)$ consisting of complexes whose Nisnevich cohomology sheaves are homotopy invariant. (This category is denoted by $\mathbf{D M}_{\mathrm{Nis}}^{\mathrm{eff},-}(k)$ in loc. cit.). 
Similarly, $\mathbf{D} \mathbf{M}_{\mathrm{h}}^{\text {eff, }-} \subset \mathbf{D}^{-}\left(\mathbf{S h v}_{\mathrm{h}}(\mathbf{S c h})\right)$ denotes the full subcategory, whose objects have homotopy invariant cohomology h-sheaves. This category is called category of effective motivic complexes without transfers.

We write $\mathbf{D} \mathbf{M}_{\mathrm{h}}^{\mathrm{eff},-}(\mathbb{Q})$ and $\mathbf{D} \mathbf{M}_{\mathrm{Nis}, \mathrm{efr}}^{\mathrm{eff}}(\mathbb{Q})$ for the corresponding $\mathbb{Q}$-linear categories.

Definition $3.2[15, \S 2]$ Let $\Delta^{*}$ denote the standard cosimplicial object in Sch, i.e. $\Delta^{n}=$ $\left\{\sum x_{i}=1\right\} \subset \mathbb{A}^{n+1}$. For any presheaf $F$, let $C_{*}(F)$ denote the Suslin complex $F\left(\Delta^{*} \times-\right)$, i.e. $C_{n}(F)(X)=F\left(\Delta^{n} \times X\right)$; its differentials are alternating sums of $\delta_{j}$. We get an exact functor $C_{*}: \operatorname{PSh}(\mathbf{S c h}) \rightarrow \operatorname{Com}(\mathbf{P S h}(\mathbf{S c h}))$. If $F$ is a sheaf with respect to an arbitrary (pre)topology, then $C_{*}(F)$ is a complex of sheaves as well as well.

Recall from Definition A. 2 that $\langle-\rangle$ denotes the minimal localising subcategory containing a given class of objects. For the rest of this subsection, we will use the following notation: $\mathcal{D}_{\mathrm{h}}:=\mathbf{D}^{-}\left(\mathbf{S h v}_{\mathrm{h}}(\mathbf{S c h}, \mathbb{Q})\right), \mathcal{A}_{\mathrm{h}}:=\left\langle\left\{\mathbb{Q}_{\mathrm{h}}\left(X \times \mathbb{A}^{1}\right) \rightarrow \mathbb{Q}_{\mathrm{h}}(X), X \in \mathbf{S m}\right\}\right\rangle \subset \mathcal{D}_{\mathrm{h}}$.

Remark 3.3 Morphisms whose cone is in $\mathcal{A}_{\mathrm{h}}$ are called weak $\mathbb{A}^{1}$-equivalences. Objects $L \in \mathcal{D}_{\mathrm{h}}$ are called $\mathbb{A}^{1}$-local, if for all weak $\mathbb{A}^{1}$-equivalences $K \rightarrow K^{\prime}$ the induced map $\operatorname{Hom}\left(K^{\prime}, L\right) \rightarrow \operatorname{Hom}(K, L)$ is a bijection.

Theorem 2.11 implies that $\mathbf{D M}_{\mathrm{h}}^{\mathrm{eff},-}(\mathbb{Q})$ is a triangulated subcategory of $\mathcal{D}_{\mathrm{h}}$ : For a distinguished triangle $A \rightarrow B \rightarrow C \rightarrow A[1]$ in $\mathcal{D}_{\mathrm{h}}$ with $A, B \in \mathbf{D M}_{\mathrm{h}}^{\mathrm{eff},-}$ we have that $\operatorname{Hom}_{\mathcal{D}_{\mathrm{h}}}\left(\mathbb{Q}_{\mathrm{h}}(-), A[n]\right)=\mathbb{H}_{\mathrm{h}}^{n}(-, A)$ is homotopy invariant, analogously for $B$. The long exact $\operatorname{Hom}_{\mathcal{D}_{\mathrm{h}}}$-sequence coming from this triangle shows that $C$ is $\mathbb{A}^{1}$-local as well, compare [15, Lemma 9.17.]. Hence, $C$ has homotopy invariant cohomology sheaves. The latter can be seen as in [15, Proposition 14.5] (using Theorem 2.11). As in loc. cit. one sees that bounded above complexes (of sheaves of $\mathbb{Q}$-vector spaces) with homotopy invariant cohomology sheaves are $\mathbb{A}^{1}$-local.

Proposition 3.4 With these notations there is an equivalence of categories $\mathcal{D}_{\mathrm{h}} / \mathcal{A}_{\mathrm{h}} \cong$ $\mathbf{D M}_{\mathrm{h}}^{\mathrm{eff},-}(\mathbb{Q})$ induced by a triangulated functor $\mathbf{R} C: \mathcal{D}_{\mathrm{h}} \longrightarrow \mathbf{D M}_{\mathrm{h}}^{\mathrm{eff},-}(\mathbb{Q})$, whose restriction on $\mathbf{S h v}_{\mathrm{h}}(\mathbf{S c h})$ is given by $C_{*}$.

Proof (compare [24, Proposition 3.2.3]). For brevity, we omit the specification of $\mathbb{Q}$-vector space sheaves in the notations. We show that the restriction of the projection map $\Phi$ : $\mathbf{D M}_{\mathrm{h}}^{\text {eff, }-} \subset \mathcal{D}_{\mathrm{h}} \stackrel{\text { Proj. }}{\longrightarrow} \mathcal{D}_{\mathrm{h}} / \mathcal{A}_{\mathrm{h}}$ is an equivalence of categories and that the restriction to $\mathrm{h}$ sheaves of the inverse equivalence $\Phi^{-1}$ is just $C_{*}$ :

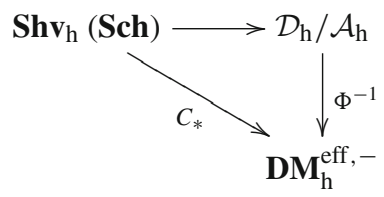

Then, we set $\mathbf{R} C:=\Phi^{-1} \circ$ Proj. According to Remark 3.3 and [16, Theorem 2.1.8], $\Phi$ is triangulated and so is $\mathbf{R} C$.

Firstly, we show that $\Phi$ is fully faithful. Secondly, we show the essential surjectivity. Both parts will use the cohomology comparison results for homotopy invariant sheaves with transfers.

$\Phi$ is fully faithful: Let $T \in \mathbf{D M}_{\mathrm{h}}^{\mathrm{eff},-}$ be arbitrary. According to Proposition $2.2,\left.T\right|_{\mathbf{S m}}$ is a complex of sheaves with transfers, which implies (Theorem 2.11) for all $X \in \mathbf{S m}, n \in \mathbb{Z}$

$$
\operatorname{Hom}_{\mathcal{D}_{\mathrm{h}}}\left(\mathbb{Q}_{\mathrm{h}}(X), T[n]\right) \cong \operatorname{Hom}_{\mathcal{D}_{\mathrm{h}}}\left(\mathbb{Q}_{\mathrm{h}}\left(X \times \mathbb{A}^{1}\right), T[n]\right) .
$$


Hence (compare [15, Lemma 9.17]) $T$ is an $\mathbb{A}^{1}$-local object in $\mathcal{D}_{\mathrm{h}}$. Then we have for all $S \in \mathcal{D}_{\mathrm{h}}: \operatorname{Hom}_{\mathcal{D}_{\mathrm{h}} / \mathcal{A}_{\mathrm{h}}}(S, T) \cong \operatorname{Hom}_{\mathcal{D}_{\mathrm{h}}}(S, T)[16$, Lemma 9.1.5].

$\Phi$ is essentially surjective: we know $K \cong \operatorname{Tot}\left(C_{*} K\right)$ in $\mathcal{D}_{\mathrm{h}} / \mathcal{A}_{\mathrm{h}}$ (see [15, Lemma 9.14], the proof without transfers is strictly parallel, as the homotopy providing the quasi-isomorphism is induced by the multiplication map $\mu: \mathbb{A}^{2} \rightarrow \mathbb{A}^{1}$ ). According to Proposition 2.2, $K$ and $\operatorname{Tot}\left(C_{*}(K)\right)$ are complexes of h-sheaves with transfers (of $\mathbb{Q}$-vector spaces). The cohomology presheaves of $\operatorname{Tot}\left(C_{*}(K)\right)$ are homotopy invariant [15, Lemma 2.17], thus the associated hsheaves of the cohomology presheaves restricted to $\mathbf{S m}$ are homotopy invariant, as well (Theorem 2.11). Using the equivalence of h-sheaves on $\mathbf{S m}$ and on Sch (see the proof of Proposition 2.2), this easily implies that the same holds without restricting to $\mathbf{S m}$. Hence $\operatorname{Tot}\left(C_{*}(K)\right) \in \mathbf{D M}_{\mathrm{h}}^{\mathrm{eff},-}$ and we are done.

Remark 3.5 If one attempts to define explicitly $\Phi^{-1}: K \mapsto \operatorname{Tot}\left(C_{*} K\right)$, one has to deal with the question, whether $C_{*}$ is an exact functor. It suffices to show the right-exactness of $C_{*}$. The presheaf cokernel $F$ of an epimorphism of h-sheaves has transfers (Proposition 2.2) and satisfies $F_{\mathrm{h}}=0$. The cohomology comparison result for the h-topology (Theorem 2.11) instead of the Nisnevich analogue [15, Theorem 13.11.] yields that the h-sheafification of $C_{*}(F)$ is exact. The h-analogue of [15, Corollary 13.13.] shows that $C_{*}$ is well-defined on $\mathcal{D}_{\mathrm{h}}$.

Lemma 3.6 The categories $\mathbf{D}^{-}\left(\mathbf{S h v}_{\mathrm{h}}(\mathbf{S c h}, \mathbb{Q})\right)$ and $\mathbf{D} \mathbf{M}_{\mathrm{h}}^{\mathrm{eff},-}(\mathbb{Q})$ are tensor triangulated categories.

Proof Starting with $X \otimes Y:=X \times_{k} Y, X, Y \in \mathbf{S c h}$, one obtains a tensor structure on $\mathbf{D}^{-}\left(\mathbf{S h v}_{\mathrm{h}}(\mathbf{S c h})\right)$ in the same manner as in [15, Corollary 8.17].

The tensor structure on $\mathcal{D}_{\mathrm{h}} / \mathcal{A}_{\mathrm{h}}$ is determined by the requirement that the canonical functor $\mathcal{D}_{\mathrm{h}} \rightarrow \mathcal{D}_{\mathrm{h}} / \mathcal{A}_{\mathrm{h}}$ is a tensor functor, compare [15, Corollary 9.4.]. Proposition 3.4, i.e. $\mathcal{D}_{\mathrm{h}} / \mathcal{A}_{\mathrm{h}} \cong$ $\mathbf{D M}_{\mathrm{h}}^{\mathrm{eff},-}(\mathbb{Q})$ yields the second assertion.

Theorem 3.7 Let $k$ be a field admitting strong resolution of singularities. Using the notations of Definition 3.1 there are natural equivalences of tensor triangulated categories

$$
\mathbf{D M}_{\mathrm{Nis}, \mathrm{tr}}^{\mathrm{eff}},(\mathbb{Q}) \cong \mathbf{D M}_{\mathrm{h}}^{\mathrm{eff},-}(\mathbb{Q}) \cong \mathbf{D M}_{\mathrm{h}}(\mathbb{Q}),
$$

where $\mathbf{D M}_{\mathrm{h}}(\mathbb{Q})$ denotes the homological category of h-sheaves (with rational coefficients) $[22, \S 4.1$.$] .$

As mentioned in the introduction, this is part of [24, Theorem 4.1.12]. We wish to give a detailed proof of it. It is essentially a corollary of the cohomology comparison result of Nisnevich and h-topology (Theorem 2.11) and the fact that h-sheafs have a unique transfer structure (Proposition 2.2).

Proof We permit ourselves to omit " $\mathbb{Q}$ " in the notations.

Let us write $\mathcal{A}_{\mathrm{Nis}, \text { tr }} \subset \mathcal{D}_{\mathrm{Nis}, \text { tr }}:=\mathbf{D}^{-}\left(\mathbf{S h v}_{\mathrm{Nis} \text {,tr }}(\mathbf{S m}, \mathbb{Q})\right)$ for the localising subcategory generated by $\mathbb{Q}_{\operatorname{tr}}\left(X \times \mathbb{A}^{1}\right) \rightarrow \mathbb{Q}_{\mathrm{tr}}(X), X \in \mathbf{S m}$. We have an equivalence of categories $\mathbf{D M}_{\mathrm{Nis}, \mathrm{tr}}^{\mathrm{eff}} \cong \mathcal{D}_{\mathrm{Nis}, \text { tr }} / \mathcal{A}_{\mathrm{Nis}, \text { tr }}$ [24, Proposition 3.2.3]. Consider the composition $(-)_{\mathrm{h}}: \mathbf{S h v}_{\mathrm{Nis}, \text { tr }}(\mathbf{S m}) \rightarrow \mathbf{S h v}_{\mathrm{Nis}}(\mathbf{S m}) \rightarrow \mathbf{S h v}_{\mathrm{h}}(\mathbf{S c h})$ of the forgetful and sheafification functor, where the latter uses the extension of h-sheaves on $\mathbf{S m}$ to ones on Sch [18, Expos§II, Théoréme 4.1]. It clearly extends to the corresponding derived categories, maps $\mathcal{A}_{\mathrm{Nis}, \text { tr }}$ to $0 \in \mathbf{D M}_{\mathrm{h}}$. It suffices to show that the complexes generating $\mathcal{A}_{\mathrm{Nis}, \text { tr }}$ are mapped to 0 , which is equivalent to $K_{X}:=\operatorname{Ker}\left(\mathbb{Q}_{\operatorname{tr}}\left(X \times \mathbb{A}^{1}\right) \rightarrow \mathbb{Q}_{\operatorname{tr}}(X)\right) \mapsto 0$, i.e. that $\left(K_{X}\right)_{\mathrm{h}}$ is contractible in the sense of [22, Definition 2.2.1]. (According to Lemma 2.1, $\mathbb{Q}_{\mathrm{tr}}(X), X \in \mathbf{S m}$ maps to $\left.\mathbb{Q}_{\mathrm{h}}(X)\right)$. The latter holds obviously, as $K_{X}=\mathbb{Q}_{\mathrm{tr}}(X) \otimes K_{\text {Spec } k}$ and $\left(K_{\text {Spec } k}\right)_{\mathrm{h}}$ is contractible 
[22, Lemma 2.2.3]. We obtain a surjective functor $\Phi: \mathcal{D}_{\mathrm{Nis}, \mathrm{tr}} / \mathcal{A}_{\mathrm{Nis}, \mathrm{tr}} \rightarrow \mathbf{D M}_{\mathrm{h}}$ (Proposition 2.2). (nota bene: We have not yet used rational coefficients).

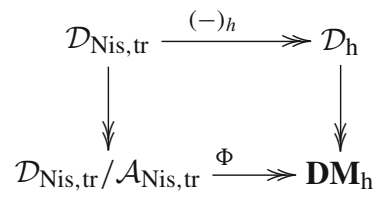

For the full faithfullness of $\Phi$ it suffices to show

$$
\operatorname{Hom}_{\mathcal{D}_{\text {Nis,tr }}}\left(\mathbb{Q}_{\text {tr }}(X), F[n]\right)=\operatorname{Hom}_{\mathbf{D M}_{\mathrm{h}}}\left(\mathbb{Q}_{\mathrm{h}}(X), F_{\mathrm{h}}[n]\right)
$$

for every $F \in \mathbf{D M}_{\mathrm{Nis}, \mathrm{tr}}^{\mathrm{eff}}$.

For brevity, we write Spec $k$ for $\mathbb{Q}_{\mathrm{h}}(\operatorname{Spec} k), X$ for $\mathbb{Q}_{\mathrm{h}}(X)$ etc. The triangle $I^{1} \rightarrow \mathbb{A}^{1} \rightarrow$ Spec $k \rightarrow I^{1}[1]$ is distinguished in $\mathcal{D}_{\mathrm{h}}$. As the category is tensor triangulated (Lemma 3.6), we obtain a long exact sequence

$$
\cdots \leftarrow \operatorname{Hom}\left(X \otimes I^{1}, F_{\mathrm{h}}[n]\right) \leftarrow \operatorname{Hom}\left(X \otimes \mathbb{A}^{1}, F_{\mathrm{h}}[n]\right) \leftarrow \operatorname{Hom}\left(X, F_{\mathrm{h}}[n]\right) \leftarrow \cdots .
$$

The two terms to the right are the $n$th hypercohomologies of $F$ in $X$ and $X \times \mathbb{A}^{1}$, respectively. They are homotopy invariant (Theorem 2.11). Hence we have $\operatorname{Hom}\left(X \otimes I^{1}, F_{\mathrm{h}}[n]\right)=0$, i.e. $F_{\mathrm{h}}$ is strictly homotopy invariant in the sense of [22, definition 2.2.8], which implies $\operatorname{Hom}_{\mathbf{D M}_{\mathrm{h}}}\left(X, F_{\mathrm{h}}[n]\right)=\operatorname{Hom}_{\mathcal{D}_{\mathrm{h}}}\left(X, F_{\mathrm{h}}[n]\right)=\mathbb{H}_{\mathrm{h}}^{n}\left(X, F_{\mathrm{h}}\right)$ [22, Proposition 2.2.9].

The equivalence $\mathbf{D} \mathbf{M}_{\mathrm{h}}^{\mathrm{eff},-} \cong \mathbf{D} \mathbf{M}_{\mathrm{h}}$ is trivial (and does not need rational coefficients).

\subsection{Geometric motives}

We now define the categories of (quasi-)geometric motives with and without transfers. The prefix "quasi" indicates that we also consider countable direct sums (i.e. disjoint unions) of schemes. Moreover, the "quasi"-categories consist of bounded above complexes.

Recall from Definition A.3 that for a class $T$ of objects of a triangulated category the notations $\langle T\rangle^{\aleph_{0}}$ (and $\langle T\rangle^{\aleph_{1}}$, respectively) mean the minimal thick subcategory containing $T$ (closed under countable existing direct sums, respectively).

Definition 3.8 The categories $\overline{\mathbb{Z S c h}}$ and $\overline{\text { SmCor }}$ are defined as the closure under countable direct sums of $\mathbb{Z}: \mathbb{Z S c h} \rightarrow \mathbf{P S h}($ Sch) (the Yoneda embedding), and similarly for $\overline{\text { SmCor. }}$ For brevity, we may write $\oplus X_{i}:=\oplus \mathbb{Z}\left(X_{i}\right)$ for a family of schemes $X_{i}$.

Definition 3.9 $[24, \S 2.1$.] Consider the class $T$ consisting of the following complexes

- $X \times \mathbb{A}^{1} \rightarrow X, X \in \mathbf{S m}$.

$-U \cap V \stackrel{\iota_{U} \oplus \iota_{V}}{\longrightarrow} U \oplus V \stackrel{\iota^{U} \oplus\left(-\iota^{V}\right)}{\longrightarrow} X$ for any open covering $X=U \cup V$ of a smooth scheme $X$.

The category of effective quasigeometric motives with transfers $\mathbf{D M}_{\overline{\mathrm{gm}}}^{\mathrm{eff}}$ is defined as follows: $\mathbf{D} \mathbf{M}_{\mathrm{gm}}^{\mathrm{eff}}:=\mathbf{K}^{-}(\overline{\mathbf{S m C o r}}) /\langle T\rangle^{\aleph_{1}}$, i.e. the localisation of $\mathbf{K}^{-}(\overline{\mathbf{S m C o r}})$ with respect to the minimal triangulated subcategory closed under countable sums containing $T$. The subcategory $\mathbf{D M}_{\mathrm{gm}}^{\text {eff }}$ consisting of $\mathbf{K}^{\mathrm{b}}$ (SmCor) is called category of effective geometric motives with transfers. (This coincides with Voevodsky's definition $\mathbf{K}^{\mathrm{b}}$ (SmCor) $/\langle T\rangle^{\aleph_{0}}$, see Theorem 3.15). 
Definition 3.10 We define the category $\mathbf{D M}_{\mathrm{gm}}^{\mathrm{eff}} \mathrm{h}$ of effective quasigeometric motives without transfers over a field $k$ : Let $T$ denote the union of the following two classes of complexes in $\mathbf{K}^{-}(\overline{\mathbb{Z} \mathbf{S c h}})$ :

$-T_{\mathbb{A}^{1}}=\left\{X \times \mathbb{A}^{1} \stackrel{p r_{1}}{\longrightarrow} X, X \in \mathbf{S c h}\right\}$.

- $T_{\mathrm{h}}=$ augmented Moore-complexes of h-coverings $U \rightarrow X, U \in \mathbf{S m}, X \in \mathbf{S c h}$ (Definition 1.2).

We define $\mathbf{D} \mathbf{M}_{\mathrm{gm}}^{\mathrm{eff}}$, to be the localisation of $\mathbf{K}^{-}(\overline{\mathbb{Z} \mathbf{S c h}})$ with respect to the $\aleph_{1}$-localising subcategory generated by $T$ :

$$
\mathbf{D M}_{\mathrm{gm}, \mathrm{h}}^{\mathrm{eff}}:=\mathbf{K}^{-}(\overline{\mathbb{Z} \mathbf{S c h}}) /\langle T\rangle^{\aleph_{1}} .
$$

The subcategory $\mathbf{D M}_{\mathrm{gm}, \mathrm{h}}^{\mathrm{eff}}$ of bounded complexes of schemes is called category of effective geometric motives without transfers. The canonical functor $\mathbf{M}_{\mathrm{gm}}: \mathbf{S c h} \rightarrow \mathbf{D M}_{\mathrm{gm}, \mathrm{h}}^{\mathrm{eff}}$ maps a scheme to its geometric motive.

If we consider rational coefficients instead of integral ones, we write $\mathbf{D M}_{\mathrm{gm}, \mathrm{h}}^{\mathrm{eff}}(\mathbb{Q})$ etc.

Remark 3.11 Note that the quasigeometric categories of motives are pseudo-abelian, as they are closed under direct sums of the form $\oplus_{i \in \mathbb{N}} A$, where $A \in \mathbf{D M}_{\overline{\mathrm{gm}}}^{\mathrm{eff}}$ [14, Lemma II.2.2.4.8.1].

Lemma 3.12 The category $\mathbf{D} \mathbf{M}_{\mathrm{gm}, \mathrm{h}}^{\mathrm{eff}}$ is a triangulated tensor category, whose tensor structure is induced by $\mathrm{M}_{\mathrm{gm}}(X) \otimes \mathrm{M}_{\mathrm{gm}}(Y)=\mathrm{M}_{\mathrm{gm}}(X \times Y)$. The subcategory $\mathbf{D M}_{\mathrm{gm}, \mathrm{h}}^{\mathrm{eff}}$ is closed under this tensor product.

Proof The tensor structure on $\mathbf{K}^{-}(\overline{\mathbb{Z} \mathbf{S c h}})$ is determined via the one on $\mathbf{S c h}(X \otimes Y:=X \times Y)$ and $\left(\oplus X_{i}\right) \otimes\left(\oplus Y_{j}\right):=\oplus_{i, j} X_{i} \otimes Y_{j}$ and $K^{*} \otimes L^{*}:=$ Tot $\left(\left(K^{n} \otimes L^{m}\right)^{n, m}\right)$. We show that for all $A \in T$ (Definition 3.10) and $B \in \mathbf{K}^{-}(\overline{\mathbb{Q S C h}})$ the tensor product $A \otimes B$ lies in $\langle T\rangle^{\aleph_{1}}$. Then $\left[14\right.$, II.2.3.4] yields the claim. The subcategory $\mathcal{B} \subset \mathbf{K}^{-}(\overline{\mathbb{Q} S \mathbf{S h}})$ containing the complexes $B$ with $A \otimes B \in\langle T\rangle^{\aleph_{1}}$ is $\aleph_{1}$-localising. Using Lemma 1.7 we reduce to the case $B \in \mathbf{S c h}$, which is obvious. The second claim is clear from the definitions.

Remark 3.13 A similar statement holds for the variants with transfers $\mathbf{D M}_{\mathrm{gm}}^{\mathrm{eff}}$ (proof as above) and $\mathbf{D M}_{\mathrm{gm}}^{\mathrm{eff}}$ [24, Proposition 2.1.3].

\subsection{Embedding theorems}

Theorem 3.14 Let $k$ admit the strong resolution of singularities. Then we have a commutative diagram of tensor-triangulated functors:

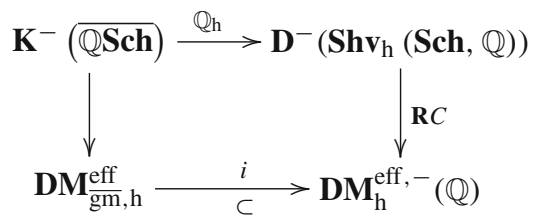

where $i$ is a full embedding of quasigeometric effective motives (Definition 3.10) into the category of effective motivic complexes (Definition 3.1). The image of $i$ is precisely the subcategory of $\aleph_{1}$-compact objects of $\mathbf{D M}_{\mathrm{h}}^{\mathrm{eff},-}(\mathbb{Q})$. The idempotent completion of the image of $\mathbf{K}^{\mathrm{b}}(\mathbb{Q} \mathbf{S c h})$ is the subcategory of compact objects in $\mathbf{D} \mathbf{M}_{\mathrm{h}}^{\mathrm{eff},-}(\mathbb{Q})$. 
Proof (compare [24, Theorem 3.2.6]). In the proof, we write $\mathcal{A}_{\mathrm{h}}:=\left\langle T_{\mathbb{A}^{1}}\right\rangle$ (see Definition 3.10) and $\mathcal{D}_{\mathrm{h}}:=\mathbf{D}^{-}\left(\mathbf{S h v}_{\mathrm{h}}(\mathbf{S c h}, \mathbb{Q})\right)$.

Existence of $i$ : By its very definition it is clear that the functor $\mathbf{R} C$ commutes with direct sums. We show that $\mathbf{R} C\left(\mathbb{Q}_{\mathrm{h}}\left(T_{\mathbb{A}^{1}}\right)\right)=0$ and $\mathbf{R} C\left(\mathbb{Q}_{\mathrm{h}}\left(T_{\mathrm{h}}\right)\right)=0$, which yields the existence of $i$. The first claim directly follows from the construction of $\mathbf{R} C$ (see Proposition 3.4, $\left.\mathcal{A}_{\mathrm{h}}=\mathbb{Q}_{\mathrm{h}}\left(T_{\mathbb{A}^{1}}\right)\right) . \mathbf{R} C: \mathcal{D} \longrightarrow \mathcal{D} / \mathcal{A}_{\mathrm{h}} \stackrel{\Phi^{-1}}{\longrightarrow} \mathbf{D M}_{\mathrm{h}}^{\text {eff, }-}$. For the second claim, we note $\mathbf{R} C\left(\mathbb{Q}_{\mathrm{h}}(Y)\right)=C_{*}\left(\mathbb{Q}_{\mathrm{h}}(Y)\right)=: C_{*}(Y)$. The inverse equivalence $\Phi^{-1}$ of Proposition 3.4 maps a complex $K$ of sheaves to the total complex $\operatorname{Tot}\left(C_{*}(K)\right)$. We have to show that the total complex of the double complex

$$
C_{*}\left(\mathcal{U}_{X} \rightarrow X \rightarrow 0\right): \cdots \longrightarrow C_{*}\left(U \times_{X} U\right) \longrightarrow C_{*}(U) \longrightarrow C_{*}(X) \longrightarrow 0
$$

is exact for any h-covering $U \rightarrow X, X \in \mathbf{S c h}$. But $C_{*}\left(\mathcal{U}_{X} \rightarrow X \rightarrow 0\right)$ is exact in view of the exactness of $C_{*}$ (see the proof of Proposition 3.4) and Lemma 1.3. As Tot preserves quasi-isomorphisms, we are done.

Full faithfulness of $i$ : Let us denote $\mathcal{D}^{\prime}:=\mathbf{D}^{-}(\mathbf{P S h}(\mathbf{S c h}, \mathbb{Q}))$. Consider the following diagram

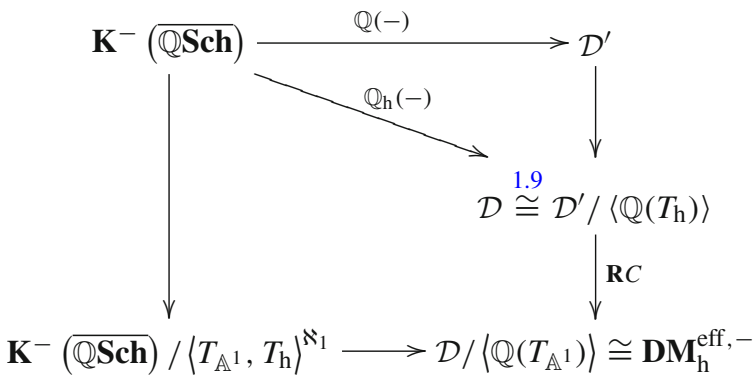

The functor $\mathbb{Q}: \mathbf{K}^{-}(\overline{\mathbb{Q S C h}}) \longrightarrow \mathcal{D}^{\prime}$ is a full embedding [26, Corollary 10.4.7] as the presheaves $\oplus_{i} \mathbb{Q}\left(X_{i}\right)$ are direct sums of projective objects, thus projective. Proposition A.5 precisely grants the extension of the embedding $\mathbf{K}^{-}(\overline{\mathbb{Q} \mathbf{S c h}}) \subset \mathcal{D}^{\prime}$ to the categories in question and the asserted compacity statements.

$i$ is tensor-triangulated: The functors $\mathbf{R} C$ and $\mathbb{Q}_{\mathrm{h}}$ are tensor functors by definition. It is clear that $\mathbb{Q}_{\mathrm{h}}$ is tensor-triangulated. In order to see that $\mathbf{R} C$ is tensor-triangulated, it suffices to remark that the projection $\mathcal{D} \rightarrow \mathcal{D} /\left\langle\mathbb{Q}_{\mathrm{h}}\left(T_{\mathbb{A}^{1}}\right)\right\rangle$ is tensor-triangulated. Hence, $\mathbf{R} C \circ \mathbb{Q}_{\mathrm{h}}$ is tensor-triangulated, which yields the claim.

\section{Theorem 3.15 There is a full embedding}

$$
i_{\text {tr }}: \mathbf{D} \mathbf{M}_{\mathrm{gm}}^{\mathrm{eff}} \subset \mathbf{D M}_{\mathrm{Nis}, \mathrm{tr}}^{\mathrm{eff},-}
$$

Similarly to the theorem above, its essential image is exactly the subcategory of $\aleph_{1}$-compact objects and the idempotent completion of $i_{\mathrm{tr}}\left(\mathbf{K}^{\mathrm{b}}(\mathbf{S m C o r})\right)$ are the compact objects of $\mathbf{D M}_{\text {Nis, }, \text { r }}^{\text {eff }}$.

Proof The proof of [24, Theorem 3.2.6] generalizes to the quasi-geometric situation (using the $\aleph_{1}$-compacity arguments as in Theorem 3.14 , Remark A.6). 


\section{Reinterpretation of geometric motives}

We are now ready to gather the results of the preceding sections in order to achieve the main result of the present paper:

Theorem 4.1 Let $k$ be a field admitting strong resolution of singularities. Then there is an equivalence of tensor triangulated categories between the categories of effective geometric motives (with rational coefficients) with and without transfers, respectively (Definitions 3.9, 3.10):

$$
\mathbf{D M}_{\mathrm{gm}, \mathrm{h}}^{\mathrm{eff}}(\mathbb{Q}) \cong \mathbf{D M}_{\mathrm{gm}}^{\mathrm{eff}}(\mathbb{Q}) .
$$

The same is true for the quasigeometric categories:

$$
\mathbf{D M}_{\overline{\mathrm{gm}}, \mathrm{h}}^{\mathrm{eff}}(\mathbb{Q}) \cong \mathbf{D M}_{\overline{\mathrm{gm}}}^{\mathrm{eff}}(\mathbb{Q}) .
$$

The theorem simplifies the handling of geometric motives insofar as one does not have to work with correspondences. Moreover, singular schemes are contained in the category in a natural manner. Last but not least, the localization with respect to the h-topology permits greater flexibility in the manipulation of objects. The price for this flexibility is the loss of torsion information.

Proof We embed the categories of effective (quasi)-geometric motives into the categories of motivic complexes, see Theorems 3.14, 3.15. Either in the case with and without transfers, these are exactly the $\left(\aleph_{1}\right)$-compact objects (Definition A.3, Proposition A.5). The equivalence $\mathbf{D M}_{\mathrm{h}}^{\mathrm{eff},-}(\mathbb{Q}) \cong \mathbf{D} \mathbf{M}_{\mathrm{Nis}, \mathrm{tr}}^{\mathrm{eff},-}(\mathbb{Q})$ (Theorem 3.7) yields the assertion.

Remark 4.2 An attempt to prove an analogous statement for a category of geometric motives defined by inverting only smooth h-hypercoverings fails, because the Moore complex of a hypercovering is in general not exact at the level of presheaves.

At first glance, the equivalence of the geometric categories, i.e. bounded complexes might be surprising, as a non-smooth scheme is ad hoc only replaced by an unbounded smooth hhypercovering. We describe a bounded complexes of smooth schemes isomorphic to a scheme $X$ : there is a proper surjective morphism $p: \tilde{X} \rightarrow X$, which is an isomorphism outside some closed subset $Z \subsetneq X$ (strong resolution of singularities). We obtain a distinguished triangle in $\mathbf{D M}=\frac{\mathrm{eff}}{\mathrm{gm}}$ of the form

$$
\mathrm{M}_{\mathrm{gm}}\left(p^{-1}(Z)\right) \rightarrow \mathrm{M}_{\mathrm{gm}}(Z) \oplus \mathrm{M}_{\mathrm{gm}}(\tilde{X}) \rightarrow \mathrm{M}_{\mathrm{gm}}(X) \rightarrow \mathrm{M}_{\mathrm{gm}}\left(p^{-1}(Z)\right)[1]
$$

[22, Proposition 4.1.5]. Inductively applying this resolution technique to $Z$ and $p^{-1}(Z)$ one constructs a so-called hypercube of schemes, one of whose vertices is $X$, the other vertices are smooth. According to the above exact triangle, the motive of $X$ is isomorphic to the total complex of the rest of the hypercube. The induction stops after at most $\operatorname{dim} X$ steps. See [7, especially Theorem 2.15] for the construction of cubical hyperresolutions.

In the sequel, we will draw some corollaries of the theorem. We will always assume that $k$ admits strong resolution of singularities and we will work with rational coefficients.

Corollary 4.3 If $A, B$ are closed subschemes of a scheme $X$, such that $A \cup B=X$, then there is a distinguished triangle in $\mathbf{D} \mathbf{M}_{\mathrm{gm}}^{\mathrm{eff}}$ of the following form:

$$
\mathrm{M}_{\mathrm{gm}}\left(A \times_{X} B\right) \rightarrow \mathrm{M}_{\mathrm{gm}}(A) \oplus \mathrm{M}_{\mathrm{gm}}(B) \rightarrow \mathrm{M}_{\mathrm{gm}}(X) \rightarrow \mathrm{M}_{\mathrm{gm}}\left(A \times_{X} B\right)[1] .
$$

In particular, as a motive, a scheme is isomorphic to the associated reduced scheme: $\mathrm{M}_{\mathrm{gm}}\left(X_{\mathrm{red}}\right) \cong \mathrm{M}_{\mathrm{gm}}(X)$. 
Proof This follows from the fact that $A \sqcup B \rightarrow X$ is a qfh-covering and Lemma 1.4.

Definition 4.4 Inverting the Tate-Object $\mathbb{Q}(1)$ in the category of effective geometric motives (with transfers), Voevodsky defines the category of geometric motives, which we denote by $\mathbf{D M}_{\mathrm{gm}}[24, \S 2.1$, page 192]. Strictly parallely, we define the category of geometric motives (without transfers) $\mathbf{D} \mathbf{M}_{\mathrm{gm}, \mathrm{h}}$ and their quasi-geometric analogs $\mathbf{D} \mathbf{M}_{\overline{\mathrm{gm}}, \mathrm{h}}$ and $\mathbf{D} \mathbf{M}_{\overline{\mathrm{gm}}}$.

Theorem 4.5 The above equivalence of effective geometric motives induces an equivalence of geometric motives with and without transfers (with rational coefficients):

$$
\mathbf{D M}_{\mathrm{gm}, \mathrm{h}}(\mathbb{Q}) \cong \mathbf{D M}_{\mathrm{gm}}(\mathbb{Q}) .
$$

Moreover, the canonical functor $\mathbf{D M}_{\mathrm{gm}, \mathrm{h}}^{\mathrm{eff}}(\mathbb{Q}) \rightarrow \mathbf{D M}_{\mathrm{gm}, \mathrm{h}}(\mathbb{Q})$ is a full embedding. These statements are also true for the quasigeometrical categories.

Proof Under the equivalence of tensor categories $\mathbf{D M}_{\mathrm{gm}, \mathrm{h}}^{\mathrm{eff}}(\mathbb{Q}) \cong \mathbf{D M}_{\mathrm{gm}}^{\mathrm{eff}}(\mathbb{Q}), \mathbb{Q}(n) \in$ $\mathbf{D M}_{\mathrm{gm}, \mathrm{h}}^{\mathrm{eff}}(\mathbb{Q})$ maps to $\mathbb{Q}(n) \in \mathbf{D M}_{\mathrm{gm}}^{\mathrm{eff}}(\mathbb{Q})$ (note that the equivalence restricted to complexes of smooth schemes is the identity). So, the first statement in either the geometric or the quasigeometric case is clear from Theorem 4.1. The second statement is then a reformulation of [24, Theorem 4.3.1] in the geometric case. The quasi-geometric case follows from the proof of loc. cit. and the fact that $\otimes$ commutes with $\oplus$.

Another consequence is a conceptual understanding of realizations of motives, similar to results of Huber [12, Theorem 2.1.6.]:

Corollary 4.6 Let $\mathcal{A}$ be any $\mathbb{Q}$-linear abelian category. Let $R: \mathbb{Q S c h} \rightarrow \mathbf{D}(\mathcal{A})$ be a functor mapping Čech nerves of h-coverings and $\mathbb{A}^{1}$-complexes (see Definition 3.10 ) to complexes quasi-isomorphic to zero. Then $R$ extends to a functor $\mathbf{D M}_{\mathrm{gm}, \mathrm{h}}^{\mathrm{eff}} \rightarrow \mathbf{D}(\mathcal{A})$. If furthermore $\mathcal{A}$ is a tensor category and tensoring in $\mathcal{A}$ with $R(\mathbb{Q}(1))$ is an equivalence of categories, $R$ extends to a functor $\mathbf{D} \mathbf{M}_{\overline{\mathrm{gm}}, \mathrm{h}} \rightarrow \mathbf{D}(\mathcal{A})$. For example, singular realization of varieties factors over geometric motives.

Acknowledgments I would like to thank Annette Huber for her constant (in)valuable support while I was writing this paper.

\section{Appendix A: Compact objects in categories of bounded above complexes}

In order to motivate the somewhat lengthy definition of $\aleph_{1}$-compact objects, we first outline the application of these notions. It turns out that the abstract characterization of complexes of schemes in terms of compacity notions makes the proof of Theorem 4.1 very simple and formal. Consider the following special case of a theorem of Neeman [16, Theorem 4.4.9], which is (tacitly) used in the proof of the embedding theorem of Voevodsky [24, Theorem 3.2.6]:

Proposition A.1 The full embedding $\mathbf{K}^{\mathrm{b}}(\mathbb{Z} \mathbf{S c h}) \subset \mathbf{D}(\mathbf{P S h}(\mathbf{S c h}))$ identifies the former category with the subcategory of compact objects of the latter category. Localizing with respect to some compact objects, for example Mayer-Vietoris complexes $U \cap V \rightarrow U \oplus V \rightarrow U \cup V$ the above embedding extends to an embedding

$$
\mathbf{K}^{\mathrm{b}}(\mathbb{Z} \mathbf{S c h}) / M .-V . \subset \mathbf{D}(\mathbf{P S h}(\mathbf{S c h})) / M .-V .
$$


Note that the right hand side is just $\mathbf{D}\left(\mathbf{S h} \mathbf{v}_{\text {Zar }}(\mathbf{S c h})\right)$. Instead of the Zariski topology, we want to invert the h-topology. In this setting, we cannot replace the unbounded Moore complexes of h-coverings by some bounded complexes. Hence we are looking for a similar statement for $\mathbf{K}^{-}$( $\left.\mathbb{Z} \mathbf{S c h}\right)$ instead of $\mathbf{K}^{\mathrm{b}}(\mathbb{Z} \mathbf{S c h})$. In order to apply the aforementioned theorem of Neeman, we enlarge $\mathbf{K}^{-}$( $\mathbb{Z} \mathbf{S c h}$ ) insofar as we add countable existing direct sums; we arrive at $\mathbf{K}^{-}(\overline{\mathbb{Z S c h}})$, which is precisely the subcategory of so-called $\aleph_{1}$-compact objects (see below). Then a similar statement as above holds (see Proposition A.5 for the precise statement).

Neeman theorem handles the case that the larger category is closed under arbitrary direct sums. So, we will have to make some minor modifications to Neeman's definitions in order to adapt them to bounded above categories like $\mathbf{D}^{-}(\mathbf{P S h}(\mathbf{S c h}))$. The idea is to add boundedness conditions in all definitions and to check that the proof of [16, Theorem 4.4.9] remains valid.

In the following definitions, let $\beta$ be an infinite cardinal, let $\mathcal{A}$ be an abelian category having all direct sums, e.g. $\mathcal{A}=\mathbf{P S h}(\mathbf{S c h})$, and $\mathcal{D}:=\mathbf{D}^{-}(\mathcal{A})$.

Definition A.2 A triangulated subcategory $\mathcal{S} \subset \mathcal{D}$ is called $\beta$-localising, if it is thick and closed under direct sums of cardinality $<\beta$ that exist in $\mathcal{D}$. The smallest $\beta$-localising subcategory containing a class of objects $S$ will be denoted $\langle S\rangle\left(\langle S\rangle^{\beta}\right.$, respectively). Hence $\langle S\rangle^{\aleph_{0}}$ is the minimal thick subcategory containing $S$ and $\langle S\rangle^{\aleph_{1}}$ is the minimal subcategory closed under countable existing direct sums. We only need these two cases.

To any complex $C^{*} \in \mathcal{D}$, we associate an integer $|C|:=\max \left\{n, C^{n} \neq 0\right\}$. (Recall that our complexes have differentials of degree +1 ). Let $\left\{X_{\lambda}, \lambda \in \Lambda\right\}$ be a set of objects in $\mathcal{D}$. It is called bounded if $\sup \left\{\left|X_{\lambda}\right|, \lambda \in \Lambda\right\}$ is finite, which implies that the direct $\operatorname{sum} \oplus X_{\lambda}$ exists (in $\mathcal{D})$. We make the following definitions (note that working in the category $\mathbf{D}(\mathcal{A})$ and dropping the boundedness conditions, we exactly arrive at Neeman's definitions $[16, \S \S 3,4]$ ).

Definition A.3 - A class $S$ of objects of $\mathcal{D}$ is called $\beta$-perfect if it contains the zero object and the following conditions hold:

1. Let $\left\{X_{\lambda}, \lambda \in \Lambda\right\}$ be a bounded collection of cardinality $<\beta$ of objects in $\mathcal{D}$. Any morphism $k \rightarrow \oplus X_{\lambda}$ with $k \in S$ factors as $k \rightarrow \oplus k_{\lambda} \stackrel{\oplus f_{\lambda}}{\longrightarrow} \oplus X_{\lambda}$, where $k_{\lambda} \in S$. Furthermore, if $\Lambda$ is infinite, we also require $\sup \left|k_{\lambda}\right| \leq \sup \left|X_{\lambda}\right|$ (which ensures that $\oplus k_{\lambda}$ exists).

2. If any such morphism $k \rightarrow \oplus k_{\lambda} \stackrel{\oplus f_{\lambda}}{\rightarrow} \oplus X_{\lambda}$ vanishes, then there are objects $l_{\lambda} \in S$ such that $f_{\lambda}$ is factorized by $k_{\lambda} \rightarrow l_{\lambda} \rightarrow X_{\lambda}$ and $k \rightarrow \oplus k_{\lambda} \rightarrow \oplus l_{\lambda}$ vanishes. Again, if $\Lambda$ is an infinite set, we require $\sup \left|l_{\lambda}\right| \leq \sup \left|X_{\lambda}\right|$.

- There is a maximal $\beta$-perfect class of objects, contained in $\mathcal{S}$ [16, Corollary 3.3.10]. It will be denoted $\mathcal{S}_{\beta}$.

- An object $k \in \mathcal{D}$ is called $\beta$-small, if any morphism $k \rightarrow \oplus_{\lambda \in \Lambda} X_{\lambda}$, where the family $X_{\lambda}$ is supposed to be bounded, factors as $k \rightarrow \oplus_{\lambda \in \Lambda^{\prime}} X_{\lambda} \rightarrow \oplus_{\lambda \in \Lambda} X_{\lambda}$, where $\Lambda^{\prime} \subset \Lambda$ is a subset of cardinality $<\beta$. The full subcategory of $\mathcal{D}$ consisting of $\beta$-small objects is denoted $\mathcal{D}^{(\beta)}$.

- The subcategory of $\beta$-compact objects in $\mathcal{D}$ is defined by $\mathcal{D}^{\beta}:=\left\{\mathcal{D}^{(\beta)}\right\}_{\beta}$. If $\beta=\aleph_{0}$, we will usually speak of compact objects.

Lemma A.4 The compact objects of $\mathcal{D}^{\prime}:=\mathbf{D}^{-}(\mathbf{P S h}(\mathbf{S c h}))$ are given by the subcategory (under the Yoneda embedding) $\mathbf{K}^{\mathrm{b}}$ (Z्Sch) of bounded complexes of schemes. The $\aleph_{1}$-compact objects of $\mathcal{D}^{\prime}$ are precisely given by bounded above complexes of countable direct products of schemes, i.e. $\mathbf{K}^{-}(\overline{\mathbb{Z} \mathbf{S c h}})$. The same is true for $\mathcal{D}:=\mathbf{D}^{-}\left(\mathbf{S h v}_{\mathrm{h}}(\mathbf{S c h})\right)$. 
Proof A scheme, considered as an object of $\mathcal{D}^{\prime}$ under the Yoneda embedding, is clearly $\aleph_{0^{-}}$ small (i.e. $\operatorname{Hom}_{\mathcal{D}^{\prime}}(\mathbb{Z}(X),-)$ ) commutes with direct sums). The same is true in the category $\mathcal{D}$. In fact, this holds for any topology $t$ whose coverings are open morphisms: by convention our schemes are quasi-compact, so every covering has a finite subcovering, which implies that the direct sum of a set of $t$-sheaves $F_{\lambda}$ is given by $X \mapsto \oplus F_{\lambda}(X)$. Via induction on the length of a complex one sees that a bounded complex of schemes is $\aleph_{0}$-small. All $\aleph_{0}$-small objects are $\left(\aleph_{0}\right.$-)compact [16, Example 3.3.16]. As the subcategory of $\beta$-compact objects is $\beta$-localising for a regular cardinal $\beta$ (such as $\aleph_{0}$ or $\aleph_{1}$ ) [16, Lemma 4.2.5] countable direct sums of schemes are $\aleph_{1}$-compact. Bounded above complexes of such objects are $\aleph_{1}$-compact as well (Lemma 1.7). We saw in Lemma 1.8 that $\left\langle\mathbf{K}^{\mathrm{b}}(\mathbb{Z} \mathbf{S c h})\right\rangle=\mathcal{D}^{\prime}$, so [16, Lemma 4.4.5] shows $\mathbf{K}^{\mathrm{b}}(\mathbb{Z} \mathbf{S c h})=\mathcal{D}^{\aleph_{0}}$ and $\mathbf{K}^{-}(\overline{\mathbb{Z} \mathbf{S c h}})=\mathcal{D}^{\aleph_{1}}$ and similarly for $\mathcal{D}$.

Proposition A.5 We write $T_{\mathrm{h}}:=\left\{\mathbb{Q}\left(\mathcal{U}_{X}\right) \rightarrow \mathbb{Q}(X), U \rightarrow\right.$ Xan h-covering $\}$ (see Definition 1.2) and $T_{\mathbb{A}^{1}}=\left\{\mathbb{Q}\left(X \times \mathbb{A}^{1}\right) \rightarrow \mathbb{Q}(X), X \in \mathbf{S m}\right\}$.

The natural functor $\mathbf{K}^{-}(\overline{\mathbb{Q S c h}}) /\left\langle T_{\mathrm{h}}\right\rangle^{\aleph_{1}} \rightarrow \mathcal{D}:=\mathbf{D}^{-}\left(\mathbf{S h v}_{\mathrm{h}}(\mathbf{S c h}, \mathbb{Q})\right)$ is a full embedding whose image consists of the $\aleph_{1}$-compact objects of $\mathcal{D}$.

The natural functor

$$
\mathbf{K}^{-}(\overline{\mathbb{Q} \mathbf{S c h}}) /\left\langle T_{\mathrm{h}}, T_{\mathbb{A}^{1}}\right\rangle^{\aleph_{1}} \rightarrow \mathcal{D} /\left\langle T_{\mathbb{A}^{1}}\right\rangle
$$

is a full embedding. Its image consists of the $\aleph_{1}$-compact objects of $\mathcal{D}$. Moreover, the idempotent completion of the image of bounded complexes of schemes are precisely the compact objects of $\mathcal{D}$.

Proof For the convenience of the reader, we indicate the necessary changes to the proof of [16, Theorem 4.4.9]: The second part of the proof [16, Lemma 3.3.3] does not work, but this lemma is only applied to triangulated subcategories, so the second part is not needed in view of [16, Lemma 3.3.5]. [16, Lemma 3.3.7] only holds for $\alpha \leq \beta$ or $\beta>\aleph_{0}$ in the notations of loc. cit. This lemma is only used in [16, Theorem 3.3.9], which is only needed with $\alpha=\beta$. [16, Theorem 4.3.3] has to be stated as follows: Let $\beta$ be a regular cardinal, $S$ a class of objects in $\mathcal{D}^{\beta}$. Let $f: x \rightarrow z$ be a morphism with $x \in \mathcal{D}^{\beta}$ and $z \in\langle S\rangle$. Then there is an object $y \in\langle S\rangle^{\beta}$, such that $|y| \leq|z|$ and $f$ factors as $x \rightarrow y \rightarrow z$. Its proof is the same as in loc. cit. This sharper statement is necessary to prove [16, Lemma 4.4.8] in our situation. The proof of the fact that localisation preserves direct sums [16, Lemma 3.2.10] does a priori not work in general, but when we localise with respect to h-coverings, the claim holds, as schemes are quasi-compact (cf. the proof of Lemma A.4). When we localise with respect to $\mathbb{A}^{1}$-complexes, the assertion is true because of Proposition 3.4.

The first claim of the proposition is now precisely the statement of [16, Theorem 4.4.9]: $\mathbf{K}^{-}(\overline{\mathbb{Z S c h}})$ are the $\aleph_{1}$-compact objects in $\mathbf{D}^{-}(\mathbf{P S h}(\mathbf{S c h}))$, which is generated by these complexes and $\mathcal{D}$ is the localisation of the $\mathcal{D}^{\prime}$ with respect to $\left\langle T_{\mathrm{h}}\right\rangle$ (Lemma 1.9). The second claim follows from the first one (using [16, Theorem 4.4.9] again).

Remark A.6 The above proposition also holds for Nisnevich sheaves with transfers. Indeed, by [24, Proposition 3.1.9] $\operatorname{Hom}_{\mathbf{D}^{-}\left(\mathbf{S h v}_{\mathrm{Nis}, \mathrm{tr}}(\mathbf{S m})\right)}(X, K[i])=\mathbb{H}_{\mathrm{Nis}}^{i}(X, K)$, Nisnevich hypercohomology commutes with direct sums and Proposition 3.4 is replaced by its predecessor [24, Proposition 3.2.3].

\section{References}

1. Abramovich, D., Karu, K., Matsuki, K., Wlodarczyk, J.: Torification and factorization of birational maps. J. Am. Math. Soc. 15(3), 531-572 (2002) 
2. Bonavero, L.: Factorisation faible des applications birationnelles (d'après Abramovich, Karu, Matsuki, Wlodarczyk et Morelli). Astérisque 282, Exp. No. 880, vii, 1-37. Séminaire Bourbaki, vol. 2000/2001 (2002)

3. de Jong, A.J.: Smoothness, semi-stability and alterations. Inst. Hautes Études Sci. Publ. Math. 83, 51-93 (1996)

4. Fulton, W.: Intersection theory, 2nd edn. Ergebnisse der Mathematik und ihrer Grenzgebiete. 3. Folge. A Series of Modern Surveys in Mathematics, vol. 2. Springer, Berlin (1998)

5. Friedlander, E.M., Voevodsky, V.: Bivariant cycle cohomology. Cycles, Transfers, and Motivic Homology Theories. Ann. of Math. Stud., vol. 143. pp. 138-187. Princeton University Press, Princeton (2000)

6. Goerss, P.G., Jardine, J.F.: Simplicial homotopy theory. Progress in Mathematics, vol. 174. Birkhäuser Verlag, Basel (1999)

7. Guillén, F., Navarro Aznar, V., Pascual Gainza, P., Puerta, F.: Hyperrésolutions cubiques et descente cohomologique. Lecture Notes in Mathematics, vol. 1335. Springer, Berlin (1988)

8. Grothendieck, A.: Éléments de géométrie algébrique. IV. Étude locale des schémas et des morphismes de schémas IV. Inst. Hautes Études Sci. Publ. Math. (1964-1967), no. 20, 24, 28, 32, 361

9. Gillet, H., Soulé, C.: Descent, motives and $K$-theory. J. Reine Angew. Math. 478, 127-176 (1996)

10. Hironaka, H.: Resolution of singularities of an algebraic variety over a field of characteristic zero. I, II. Ann. Math. 79(2), 109-203, 205-326 (1964)

11. Hironaka, H.: Flattening theorem in complex-analytic geometry. Am. J. Math. 97, 503-547 (1975)

12. Huber, A.: Realization of Voevodsky's motives. J. Algebraic Geom. 9(4), 755-799 (2000)

13. Ivorra, F.: Réalisation 1-adique des motifs mixtes. Thesis, Université Paris 6. http://www.math.uiuc.edu/ K-theory/0762 (2005)

14. Levine, M.: Mixed motives. Mathematical Surveys and Monographs, vol. 57. American Mathematical Society, Providence (1998)

15. Mazza, C., Voevodsky, V., Weibel, C.: Lectures in Motivic Cohomology. Preprint. http://www.math. rutgers.edu/ weibel/motiviclectures.html

16. Neeman, A.: Triangulated categories. Annals of Mathematics Studies, vol. 148. Princeton University Press, Princeton (2001)

17. Raynaud, M., Gruson, L.: Critères de platitude et de projectivité. Techniques de "platification" d'un module. Invent. Math. 13, 1-89 (1971)

18. Théorie des topos et cohomologie étale des schémas. Tome 1: Théorie des topos. Séminaire de Géométrie Algébrique du Bois-Marie 1963-1964 (SGA 4), Dirigé par M. Artin, A. Grothendieck, et J. L. Verdier. Avec la collaboration de N. Bourbaki, P. Deligne et B. Saint-Donat, Lecture Notes in Mathematics, vol. 269, pp. xix+525. Springer-Verlag, Berlin (1972)

19. Théorie des topos et cohomologie étale des schémas. Tome 2: Séminaire de Géométrie Algébrique du BoisMarie 1963-1964 (SGA 4), Dirigé par M. Artin, A. Grothendieck et J. L. Verdier. Avec la collaboration de N. Bourbaki, P. Deligne et B. Saint-Donat, Lecture Notes in Mathematics, vol. 270, pp. iv+418. Springer-Verlag, Berlin (1972)

20. Suslin, A., Voevodsky, V.: Singular homology of abstract algebraic varieties. Invent. Math. 123(1), 6194 (1996)

21. Suslin, A., Voevodsky, V.: Relative cycles and Chow sheaves. Cycles, Transfers, and Motivic Homology Theories. Ann. of Math. Stud., vol. 143, pp. 10-86. Princeton University Press, Princeton (2000)

22. Voevodsky, V.: Homology of schemes I. Selecta Math. 2(1), 111-153 (1996)

23. Voevodsky, V.: Cohomological theory of presheaves with transfers. Cycles, Transfers, and Motivic Homology Theories. Ann. of Math. Stud., vol. 143, pp. 87-137. Princeton University Press, Princeton (2000)

24. Voevodsky, V.: Triangulated categories of motives over a field. Cycles, Transfers, and Motivic Homology Theories. Ann. of Math. Stud., vol. 143, pp. 188-238. Princeton University Press, Princeton (2000)

25. Voevodsky, V., Suslin, A., Friedlander, E.M.: Cycles, Transfers, and Motivic Homology Theories. Annals of Mathematics Studies, vol. 143. Princeton University Press, Princeton (2000)

26. Weibel, C.A.: An introduction to homological algebra. Cambridge Studies in Advanced Mathematics, vol.38. Cambridge University Press, Cambridge (1994) 